\title{
$X$-ray variability in a complete sample of Soft X-ray selected $\mathrm{AGN}^{\star}$
}

\author{
D. Grupe ${ }^{1, \star \star}$, H.-C. Thomas ${ }^{2}$, and K. Beuermann ${ }^{1,3}$ \\ 1 MPI für extraterrestrische Physik, Postfach 1312, 85741 Garching, Germany \\ 2 MPI für Astrophysik, Karl-Schwarzschild-Str. 1, 85741 Garching, Germany \\ 3 Universitäts-Sternwarte, Geismarlandstr. 11, 37083 Göttingen, Germany
}

Received 14 August 2000 / Accepted 24 November 2000

\begin{abstract}
We present ROSAT All-Sky Survey and ROSAT pointed observations (PSPC and HRI) of a complete sample of 113 bright soft X-ray AGN selected from the ROSAT Bright Source Catalog. We compare these observations in order to search for extreme cases of flux and spectral X-ray variability - X-ray transient AGN. Three definite transients and one transient candidate are found. The other sources show amplitude variations typically by factors of 2-3 on timescales of years. We found that the variability strength on timescales of days is a function of the steepness of the X-ray spectrum: steeper X-ray objects show stronger variability than flat X-ray spectrum sources. We also present new HRI measurements of our extreme X-ray transients IC 3599 and WPVS007. We discuss possible models to explain the X-ray transience and the variabilities observed in the non-transient sources.
\end{abstract}

Key words. accretion, accretion disks - galaxies: active - galaxies: nuclei of - galaxies: Seyfert - quasars: general

\section{Introduction}

The optical-UV-soft X-ray bump has turned out to be a common property of most Narrow-Line Seyfert 1 (NLS1) type Active Galactic Nuclei (AGN). With ROSAT's (Trümper 1983) Position Sensitive Proportional Counter (PSPC, Pfeffermann et al. 1986) with its spectral sensitivity to energies below $0.5 \mathrm{keV}$, numerous AGN have been found that show a soft X-ray excess, commonly believed to be the high energy part of the "Big Blue/UV Bump". This bump emission is thought to be produced by an accretion disk that surrounds the central black hole. The soft X-ray emission is explained by Compton scattering of thermal UV disk photons by a hot electron layer above the disk. The UV photon spectrum is shifted further into the soft $\mathrm{X}$-ray range as the engine accretes closer to its Eddington accretion rate (e.g. Ross et al. 1992). Before ROSAT, a study of soft X-ray selected AGN had to rely on serendipitous observations, notably with the EINSTEIN Image Proportional Counter (Córdova et al. 1992; Puchnarewicz et al. 1992)

Send offprint requests to: D. Grupe,

e-mail: dgrupe@xray.mpe.mpg.de

* Based in part on observations at the European Southern Observatory La Silla (Chile) with the $2.2 \mathrm{~m}$ telescope of the Max-Planck-Society during MPG and ESO time and the ESO $1.52 \mathrm{~m}$ telescope.

** Guest Observer, McDonald Observatory, University of Texas at Austin.
The ROSAT All-Sky Survey (RASS, Voges et al. 1999) and later re-observations of many sources made it possible to study the long-term behaviour of AGN in X-rays. AGN can vary in three ways, either by changes in the flux (or count rate), solely by spectral changes, or a combination of both. Most common are variations in X-ray flux by factors of 2-3 on timescales of days and years (e.g. Lawrence et al. 1977; Green et al. 1993; Boller et al. 1996). However, factors of more than ten have been reported for several sources (e.g. IRAS 13224-3809, Boller et al. 1997 or PHL 1092, Brandt et al. 1999). AGN can also change the shape of their X-ray spectra. The most extreme example of such a spectral change has been reported on the Narrowline Seyfert 1 galaxy (NLS1) RX J0134.2-4258 (Grupe et al. 2000; Komossa \& Meerschweinchen 2000). It has been shown that the timescales of the variabilities found in AGN scale with luminosity (Barr \& Mushotzky 1986; Lawrence \& Papadakis 1993; Green et al. 1993). Leighly (1999a,b) has presented a comprehensive variability study on a sample of 25 AGN observed by ASCA and found a) that the variability strength is a function of the luminosity and b) that NLS1 are more variable than Broad-Line Seyfert 1s. Boller et al. (1996) found in their NLS1 study based on ROSAT data that the timescale of the variability is a function of the luminosity. Variability in X-rays can be due either to changes in the absorption column or intrinsic variability of the X-ray source. Absorption by both neutral and ionized gas has been proposed to explain the 
X-ray variability observed (see Abrassart \& Czerny 2000 and Komossa \& Meerschweinchen 2000). Intrinsic variability can be caused by e.g. changes in the accretion rate or relativistic beaming effects (e.g. Boller et al. 1997).

With the RASS a new AGN phenomenon has been established: X-ray transience. X-ray transience is the most extreme form of variability in AGN. On timescales of years, the count rates decreased by factors of more than 100 or the sources even vanished in X-rays. The first example of an X-ray transient AGN was given by Piro et al. (1988) who reported a change in the soft X-ray luminosity of the Seyfert 1 galaxy E1615+061 by two orders of magnitude on timescales of years. Transient sources are thought to accrete at high rates, close to the Eddington limit, and therefore have very soft X-ray spectra. The RASS with its sensitivity to energies down to $0.1 \mathrm{keV}$ has a high potential to find transient sources. Transience in AGN can be caused by changes of accretion disk properties (e.g. the temperature, see WPVS007, Grupe et al. 1995b), a dramatic increase of the accretion rate (e.g. in an outburst as seen in IC 3599, Brandt et al. 1995; Grupe et al. 1995a), or alternatively, changes in the absorption column in the line of sight.

Thomas et al. (1998) have presented a completely identified sample of all 397 bright soft X-ray selected, high galactic latitude RASS sources (mean PSPC count rate $\geq 0.5 \mathrm{cts} \mathrm{s}^{-1}$, hardness ratio $1(H R 1)<0.00^{1}$, and $|b|>20^{\circ}$, based on RASS II). Of these sources, 113 are AGN, which is the sample we present here. Our sample is complete for all AGN following the criteria above. BL Lac objects were excluded because of their different X-ray emission mechanism.

The task of this new paper is to compare the RASS results with measurements of ROSAT pointed observations in order to find new X-ray transient AGN. In Sect. 2 we describe the observations and data reduction. Section 3 shows the results obtained for the whole sample and lists individual X-ray transient AGN, and in Sect. 4 we discuss the results. Throughout the paper, luminosities are calculated assuming a Hubble constant of $H_{0}=75 \mathrm{~km} \mathrm{~s}^{-1} \mathrm{Mpc}^{-1}$ and a deceleration parameter of $q_{0}=0$. Spectral slopes, $\alpha$, are defined by $F \propto \nu^{-\alpha}$.

\section{Observations and data reduction}

All objects have been observed (by definition) during the RASS. The data from RASS $\mathrm{II}^{2}$ were extracted from the event files as described in Grupe et al. (1998a). Pointed PSPC and High Resolution Imager (HRI) observations were derived from the ROSAT public data archive at MPE Garching (ftp.xray.mpe.mpg.de). The source

1 The hardness ratio is defined as $H R 1=$ (hardsoft $) /($ hard+soft $)$ with soft PSPC channels $=11-41$ and hard $=52-201$.

2 The difference between RASS I and RASS II is in the detection likelihood for acceptance of a source; Voges et al. (1999). Meanwhile the RASS III has been released.
PSPC count rates were determined using a weighted exposure map in four energy channel ranges, 8-41, 42-51, 52-90, and $91-201^{3}$. Power-law spectral models were applied to the RASS as well as to the pointed PSPC data. We limit the spectral analysis to single power-law fits only because of the limited signal-to-noise ratios of the RASS observations. The absorption parameter for cold absorption $N_{\mathrm{H}}$ was either fixed to the Galactic value given by Dickey \& Lockman (1990), or left as a free parameter (see Table 4).

In cases where more than one pointed PSPC (or HRI) observation was available in the archive, we usually took the longest one in order to get better signal-to-noise ratio. HRI count rates were converted into effective PSPC count rates using the W3PIMMS program of NASA's Goddard Space Flight Center (version 2.7, 1999, http://heasarc.gsfc.nasa.gov/Tools/w3pimms.html) based on the power-law fits to the RASS data with the absorption parameter $N_{\mathrm{H}}$ fixed to the galactic value, and assuming no spectral changes between the RASS and the HRI observations. For all X-ray reduction and analysis tasks we used the EXSAS data analysis package of the MPE Garching (5th edition, Zimmermann et al. 1998).

\section{Results}

Table 1 lists the optical coordinates derived from the US Naval Observatory scans (USNO A2.0) and summarizes the count rates and hardness ratios of the RASS, pointed PSPC, and HRI observations. The X-ray positions of the objects are given in Thomas et al. (1998). The RASS count rates and HR1 given in Table 1 differ slightly from the values in the ROSAT Bright Source Catalogue (BSC, Voges et al. 1999), because while the definition of the sample is based on the BSC our analysis of the RASS event files were used for all analysis throughout the paper. In two cases (RX J1624.9+7554 and RX J2312.5-3404) the count rates are below the limit of $0.5 \mathrm{cts} \mathrm{s}^{-1}$ and in one case, IIZW136, the $H R 1>0.00$. All observations used for this publication, with their observing dates, exposure times and the ROSAT Observation Request number (ROR) of the pointed observations, are listed in Table 2.

\subsection{Flux variability}

Figure 1 compares the RASS vs. PSPC and HRI pointed observation count rates. In general, if PSPC and HRI observations were available for one source, preference was given to the PSPC observation, because RASS and PSPC pointings were performed by the same type of detector and allow, therefore, a direct comparison without any assumptions about the spectral shape that is necessary for converting the HRI observations. The plot displays that the distribution of the converted HRI count rates does not differ significantly from the PSPC data. It shows that the method of conversion is basically correct.

\footnotetext{
${ }^{3}$ One energy channel transfers to $10 \mathrm{eV}$.
} 


\begin{tabular}{|c|c|c|c|c|c|c|c|c|c|c|c|}
\hline \multirow{2}{*}{ No. } & \multirow{2}{*}{$\begin{array}{l}\alpha_{2000} \\
\mathrm{~h} \mathrm{~m} \mathrm{~s}\end{array}$} & \multirow{2}{*}{$\begin{array}{l}\delta_{2000} \\
\circ^{\prime}, " \prime\end{array}$} & \multirow{2}{*}{ Name } & \multirow{2}{*}{$z$} & \multicolumn{2}{|c|}{ RASS } & \multicolumn{2}{|c|}{ PSPC pointed } & \multicolumn{3}{|c|}{ HRI pointed } \\
\hline & & & & & $\begin{array}{c}\mathrm{CR} \\
\operatorname{cts~s}^{-1}\end{array}$ & HR1 & $\begin{array}{c}\text { CR } \\
\text { cts s }^{-1}\end{array}$ & HR1 & $\begin{array}{c}\text { expected } \\
\text { HRI CR }\end{array}$ & $\begin{array}{c}\mathrm{CR} \\
\mathrm{ctss}^{-1}\end{array}$ & $\begin{array}{c}\text { converted } \\
\text { CR }\end{array}$ \\
\hline 1 & 000619.5 & +201211 & Mkn 335 & 0.026 & $2.33 \pm 0.10$ & $-0.07 \pm 0.04$ & $2.60 \pm 0.01$ & $-0.04 \pm 0.02$ & 0.660 & $0.426 \pm 0.007$ & 1.504 \\
\hline 2 & 002500.2 & -452934 & ESO 242-G8 & 0.059 & $0.79 \pm 0.08$ & $-0.48 \pm 0.07$ & $0.47 \pm 0.01$ & $-0.43 \pm 0.09$ & 0.183 & $0.060 \pm 0.006$ & 0.260 \\
\hline 3 & 003915.8 & -511702 & WPVS 007 & 0.029 & $0.96 \pm 0.07$ & $-0.97 \pm 0.02$ & $0.002 \pm 0.001$ & 1 & 0.197 & 0.001 & 0.004 \\
\hline 4 & 005720.2 & -222257 & RX J0057.2-2223 & 0.062 & $2.72 \pm 0.10$ & $-0.58 \pm 0.03$ & $3.28 \pm 0.03$ & $-0.59 \pm 0.05$ & - & - & - \\
\hline 5 & 005837.4 & -360605 & QSO 0056-36 & 0.165 & $0.75 \pm 0.06$ & $-0.31 \pm 0.06$ & - & - & 0.178 & $0.162 \pm 0.008$ & 0.680 \\
\hline 6 & 010027.1 & -511354 & RX J0100.4-5113 & 0.062 & $1.06 \pm 0.08$ & $-0.24 \pm 0.07$ & - & - & 0.272 & $0.127 \pm 0.005$ & 0.495 \\
\hline 7 & 010538.8 & -141614 & RX J0105.6-1416 & 0.070 & $1.56 \pm 0.07$ & $-0.17 \pm 0.04$ & - & - & - & - & - \\
\hline 8 & 011730.6 & -382630 & RX J0117.5-3826 & 0.225 & $0.98 \pm 0.07$ & $-0.52 \pm 0.04$ & - & - & - & - & - \\
\hline 9 & 011935.7 & -282132 & MS 0117-28 & 0.349 & $0.60 \pm 0.06$ & $-0.72 \pm 0.05$ & $0.48 \pm 0.01$ & $-0.79 \pm 0.02$ & - & - & - \\
\hline 10 & 012806.7 & -184831 & RX J0128.1-1848 & 0.046 & $0.76 \pm 0.06$ & $-0.28 \pm 0.06$ & - & - & - & - & - \\
\hline 11 & 012910.7 & -214157 & IRAS F01267-217 & 0.093 & $0.83 \pm 0.07$ & $-0.45 \pm 0.06$ & - & - & 0.192 & $0.482 \pm 0.011$ & 2.076 \\
\hline 12 & 014822.3 & -275826 & RX J0148.3-2758 & 0.121 & $2.35 \pm 0.09$ & $-0.62 \pm 0.03$ & $1.63 \pm 0.02$ & $-0.51 \pm 0.05$ & - & - & - \\
\hline 13 & 015227.1 & -231954 & RX J0152.4-2319 & 0.113 & $1.08 \pm 0.06$ & $-0.52 \pm 0.04$ & - & - & 0.223 & $0.241 \pm 0.007$ & 1.170 \\
\hline 14 & 023005.5 & -085953 & Mkn 1044 & 0.017 & $2.23 \pm 0.10$ & $-0.06 \pm 0.04$ & $2.10 \pm 0.03$ & $-0.02 \pm 0.05$ & - & - & - \\
\hline 15 & 023437.8 & -084716 & Mkn 1048 & 0.042 & $1.24 \pm 0.08$ & $-0.05 \pm 0.06$ & $1.30 \pm 0.02$ & $+0.04 \pm 0.05$ & 0.355 & $0.106 \pm 0.008$ & 0.371 \\
\hline 16 & 031118.8 & -204619 & RX J0311.3-2046 & 0.070 & $0.91 \pm 0.08$ & $-0.08 \pm 0.07$ & - & - & - & - & - \\
\hline 17 & 031948.7 & -262712 & RX J0319.8-2627 & 0.079 & $0.52 \pm 0.07$ & $-0.52 \pm 0.09$ & $0.78 \pm 0.01$ & $-0.35 \pm 0.06$ & - & - & - \\
\hline 18 & 032315.8 & -493111 & RX J0323.2-4931 & 0.071 & $1.45 \pm 0.06$ & $-0.50 \pm 0.03$ & $0.50 \pm 0.01$ & $-0.46 \pm 0.08$ & 0.304 & $0.094 \pm 0.007$ & 0.450 \\
\hline 19 & 032502.2 & -415418 & ESO 301-G13 & 0.059 & $0.96 \pm 0.06$ & $-0.48 \pm 0.04$ & - & - & 0.219 & $0.371 \pm 0.012$ & 1.625 \\
\hline 20 & 033340.2 & -370655 & VCV0331-37 & 0.064 & $0.68 \pm 0.08$ & $-0.40 \pm 0.09$ & - & - & 0.159 & $0.142 \pm 0.007$ & 0.605 \\
\hline 21 & 034907.7 & -471104 & RX J0349.1-4711 & 0.299 & $0.53 \pm 0.06$ & $-0.70 \pm 0.05$ & - & - & 0.095 & $0.044 \pm 0.005$ & 0.247 \\
\hline 22 & 035141.7 & -402800 & Fairall 1116 & 0.059 & $1.86 \pm 0.14$ & $-0.37 \pm 0.06$ & - & - & 0.474 & $0.205 \pm 0.009$ & 0.803 \\
\hline 23 & 040501.7 & $-3711 \quad 15$ & CTS 44 & 0.055 & $3.29 \pm 0.11$ & $-0.37 \pm 0.02$ & - & - & - & - & - \\
\hline 24 & 041241.5 & -471246 & RX J0412.7-4712 & 0.132 & $0.77 \pm 0.10$ & $-0.52 \pm 0.09$ & - & - & 0.169 & $0.261 \pm 0.008$ & 1.172 \\
\hline 25 & 042600.7 & -571202 & $1 \mathrm{H} 0419+577$ & 0.104 & $3.63 \pm 0.10$ & $-0.54 \pm 0.02$ & $6.00 \pm 0.04$ & $-0.47 \pm 0.03$ & 0.795 & $0.510 \pm 0.011$ & 2.332 \\
\hline 26 & 043040.0 & -533656 & Fairall 303 & 0.040 & $1.18 \pm 0.09$ & $-0.50 \pm 0.06$ & $0.99 \pm 0.02$ & $-0.39 \pm 0.09$ & - & - & - \\
\hline 27 & 043728.2 & -471130 & RX J0437.4-4711 & 0.052 & $1.13 \pm 0.06$ & $-0.60 \pm 0.03$ & $1.35 \pm 0.02$ & $-0.51 \pm 0.05$ & 0.230 & $0.461 \pm 0.005$ & 2.255 \\
\hline 28 & 043938.7 & -531131 & RX J0439.6-5311 & 0.243 & $0.83 \pm 0.12$ & $-0.81 \pm 0.06$ & $1.44 \pm 0.02$ & $-0.79 \pm 0.02$ & - & - & - \\
\hline 29 & 044122.5 & -270820 & Н 0439-272 & 0.084 & $0.62 \pm 0.10$ & $-0.09 \pm 0.12$ & $0.77 \pm 0.01$ & $+0.09 \pm 0.07$ & - & - & - \\
\hline 30 & 061549.6 & -582606 & 1 ES 0614-584 & 0.057 & $0.59 \pm 0.04$ & $-0.18 \pm 0.03$ & - & - & - & - & - \\
\hline 31 & 085902.9 & +484609 & RX J0859.0+4846 & 0.083 & $0.70 \pm 0.06$ & $-0.18 \pm 0.07$ & - & - & - & - & - \\
\hline 32 & 090233.6 & -070004 & RX J0902.5-0700 & 0.089 & $0.62 \pm 0.06$ & $-0.22 \pm 0.07$ & - & - & - & - & - \\
\hline 33 & 092513.0 & +521712 & Mkn 110 & 0.035 & $1.65 \pm 0.08$ & $-0.21 \pm 0.04$ & $6.30 \pm 0.03$ & $-0.28 \pm 0.02$ & 0.420 & $1.519 \pm 0.008$ & 5.967 \\
\hline 34 & 095652.4 & +411522 & PG $0953+41$ & 0.234 & $0.99 \pm 0.07$ & $-0.56 \pm 0.04$ & $0.76 \pm 0.01$ & $-0.49 \pm 0.07$ & - & - & - \\
\hline 35 & 100541.9 & +433241 & RX J1005.7+4332 & 0.178 & $0.73 \pm 0.05$ & $-0.64 \pm 0.04$ & - & - & 0.143 & $0.052 \pm 0.004$ & 0.265 \\
\hline 36 & 100710.2 & +220302 & RX J1007+2203 & 0.083 & $0.62 \pm 0.06$ & $-0.09 \pm 0.06$ & - & - & - & - & - \\
\hline 37 & 101303.2 & +355124 & CBS 126 & 0.079 & $1.43 \pm 0.07$ & $-0.45 \pm 0.03$ & - & - & 0.316 & $0.055 \pm 0.005$ & 0.249 \\
\hline 38 & 101900.5 & +375241 & HS $1019+37$ & 0.135 & $0.81 \pm 0.06$ & $-0.08 \pm 0.05$ & $0.37 \pm 0.01$ & $+0.02 \pm 0.03$ & - & - & - \\
\hline 39 & 101912.6 & +635803 & Mkn 141 & 0.042 & $0.50 \pm 0.06$ & $-0.45 \pm 0.06$ & - & - & 0.108 & $0.032 \pm 0.004$ & 0.149 \\
\hline 40 & 102531.3 & +514035 & Mkn 142 & 0.045 & $1.73 \pm 0.07$ & $-0.62 \pm 0.03$ & $1.44 \pm 0.02$ & $-0.56 \pm 0.05$ & - & - & - \\
\hline
\end{tabular}




\begin{tabular}{|c|c|c|c|c|c|c|c|c|c|c|c|}
\hline \multirow{2}{*}{ No. } & \multirow[b]{2}{*}{$\begin{array}{l}\alpha_{2000} \\
\mathrm{~h} \mathrm{~m} \mathrm{~s}\end{array}$} & \multirow[b]{2}{*}{$\begin{array}{l}\delta_{2000} \\
\circ^{\prime} \prime \prime\end{array}$} & \multirow{2}{*}{ Name } & \multirow[b]{2}{*}{$z$} & \multicolumn{2}{|c|}{ RASS } & \multicolumn{2}{|c|}{ PSPC pointed } & \multicolumn{3}{|c|}{ HRI pointed } \\
\hline & & & & & $\begin{array}{c}\mathrm{CR} \\
\operatorname{cts~s}^{-1}\end{array}$ & HR1 & $\begin{array}{c}\mathrm{CR} \\
\operatorname{cts~s}^{-1}\end{array}$ & HR1 & $\begin{array}{l}\text { expected } \\
\text { HRI CR }\end{array}$ & $\begin{array}{c}\mathrm{CR} \\
\operatorname{ctss}^{-1}\end{array}$ & $\begin{array}{c}\text { converted } \\
\mathrm{CR}\end{array}$ \\
\hline 41 & 103438.6 & +393828 & RX J1034.6+3938 & 0.044 & $2.62 \pm 0.10$ & $-0.74 \pm 0.02$ & $3.53 \pm 0.03$ & $-0.71 \pm 0.04$ & - & - & - \\
\hline 42 & $1117 \quad 10.1$ & +652207 & RX J1117.1+6522 & 0.147 & $0.58 \pm 0.05$ & $-0.72 \pm 0.04$ & - & - & 0.108 & $0.060 \pm 0.007$ & 0.325 \\
\hline 43 & $11 \quad 1830.4$ & +402555 & PG $1115+407$ & 0.154 & $0.56 \pm 0.06$ & $-0.46 \pm 0.06$ & $0.32 \pm 0.01$ & $-0.51 \pm 0.10$ & 0.121 & $0.068 \pm 0.005$ & 0.315 \\
\hline 44 & 111908.7 & +211918 & Ton 1388 & 0.177 & $1.00 \pm 0.08$ & $-0.53 \pm 0.05$ & $1.05 \pm 0.01$ & $-0.46 \pm 0.03$ & 0.213 & $0.339 \pm 0.014$ & 1.594 \\
\hline 45 & 113104.8 & +685153 & EXO $1128+69$ & 0.045 & $1.59 \pm 0.06$ & $-0.45 \pm 0.03$ & - & - & - & - & - \\
\hline 46 & 113109.5 & +311406 & B2 $1128+31$ & 0.289 & $0.56 \pm 0.06$ & $-0.11 \pm 0.08$ & - & - & - & - & - \\
\hline 48 & 113913.9 & +335551 & Z $1136+3412$ & 0.033 & $0.85 \pm 0.07$ & $-0.33 \pm 0.06$ & $0.64 \pm 0.02$ & $-0.42 \pm 0.15$ & - & - & - \\
\hline 49 & 114116.2 & +215621 & Was 26 & 0.063 & $1.60 \pm 0.07$ & $-0.13 \pm 0.04$ & $0.98 \pm 0.02$ & $-0.11 \pm 0.06$ & - & - & - \\
\hline 50 & 114429.9 & +365309 & CASG 855 & 0.040 & $1.29 \pm 0.08$ & $-0.18 \pm 0.05$ & - & - & - & - & - \\
\hline 51 & $1201 \quad 14.4$ & -034041 & Mkn 1310 & 0.019 & $0.89 \pm 0.06$ & $-0.07 \pm 0.06$ & $0.26 \pm 0.01$ & $-0.06 \pm 0.16$ & - & - & - \\
\hline 52 & 120309.5 & +443150 & NGC 4051 & 0.002 & $3.62 \pm 0.11$ & $-0.47 \pm 0.02$ & $1.68 \pm 0.01$ & $-0.41 \pm 0.02$ & 0.804 & $0.489 \pm 0.014$ & 2.209 \\
\hline 53 & 120442.1 & +275412 & GQ Com & 0.165 & $0.59 \pm 0.05$ & $-0.13 \pm 0.06$ & $0.47 \pm 0.01$ & $-0.11 \pm 0.04$ & 0.158 & $0.164 \pm 0.008$ & 0.613 \\
\hline 54 & 120945.2 & +321702 & RX J1209.8+3217 & 0.145 & $0.59 \pm 0.11$ & $-0.63 \pm 0.08$ & $0.28 \pm 0.01$ & $-0.58 \pm 0.18$ & - & - & - \\
\hline 55 & 121417.7 & +140313 & PG1211+14 & 0.082 & $1.71 \pm 0.08$ & $-0.35 \pm 0.03$ & $1.05 \pm 0.02$ & $-0.20 \pm 0.06$ & - & - & - \\
\hline 56 & 121826.6 & +294846 & Mkn 766 & 0.013 & $5.35 \pm 0.13$ & $-0.03 \pm 0.02$ & $3.53 \pm 0.02$ & $-0.09 \pm 0.03$ & 1.468 & $0.384 \pm 0.002$ & 1.399 \\
\hline 58 & 123136.6 & +704414 & RX J1231.6+7044 & 0.208 & $1.08 \pm 0.05$ & $-0.20 \pm 0.04$ & - & - & 0.273 & $0.162 \pm 0.007$ & 0.642 \\
\hline 59 & 123203.6 & +200930 & Mkn 771 & 0.064 & $0.61 \pm 0.05$ & $-0.39 \pm 0.06$ & $1.32 \pm 0.02$ & $-0.26 \pm 0.05$ & - & - & - \\
\hline 60 & 123341.7 & +310103 & CBS 150 & 0.290 & $0.51 \pm 0.05$ & $-0.53 \pm 0.05$ & $0.33 \pm 0.01$ & $-0.53 \pm 0.15$ & - & - & - \\
\hline 61 & 123651.2 & +453905 & MCG+08-23- & 0.030 & $0.58 \pm 0.05$ & $-0.29 \pm 0.06$ & - & - & - & - & - \\
\hline 62 & 123741.2 & +264228 & IC 3599 & 0.021 & $4.90 \pm 0.11$ & $-0.64 \pm 0.02$ & $0.07 \pm 0.01$ & $-0.83 \pm 0.05$ & 1.247 & $0.005 \pm 0.001$ & 0.025 \\
\hline 63 & 123939.4 & -052039 & NGC 4593 & 0.009 & $3.32 \pm 0.18$ & $-0.16 \pm 0.05$ & $1.44 \pm 0.04$ & $+0.10 \pm 0.10$ & 0.897 & $1.041 \pm 0.024$ & 3.850 \\
\hline 64 & 124210.6 & +331703 & IRAS F12397+3333 & 0.044 & $0.84 \pm 0.06$ & $-0.33 \pm 0.05$ & $0.78 \pm 0.01$ & $-0.27 \pm 0.05$ & - & - & - \\
\hline 65 & 124635.2 & +022209 & PG $1244+026$ & 0.049 & $1.28 \pm 0.10$ & $-0.50 \pm 0.06$ & $1.15 \pm 0.02$ & $-0.44 \pm 0.05$ & - & - & - \\
\hline 66 & $1304 \quad 17.0$ & +020537 & RX J1304.2+0205 & 0.229 & $0.79 \pm 0.07$ & $-0.64 \pm 0.05$ & - & - & - & - & - \\
\hline 67 & 130947.0 & +081948 & PG $1307+085$ & 0.155 & $0.60 \pm 0.06$ & $-0.24 \pm 0.06$ & $0.56 \pm 0.01$ & $-0.24 \pm 0.06$ & 0.153 & $0.100 \pm 0.006$ & 0.395 \\
\hline 68 & 131422.7 & +342939 & RX J1314.3+3429 & 0.075 & $0.68 \pm 0.06$ & $-0.62 \pm 0.04$ & $0.74 \pm 0.03$ & $-0.70 \pm 0.20$ & 0.128 & $0.117 \pm 0.005$ & 0.620 \\
\hline 69 & 131957.1 & +523533 & RX J1319.9+5235 & 0.092 & $0.68 \pm 0.05$ & $-0.53 \pm 0.05$ & - & - & - & - & - \\
\hline 70 & 132349.5 & +654148 & PG $1322+659$ & 0.168 & $0.71 \pm 0.05$ & $-0.48 \pm 0.04$ & $0.54 \pm 0.01$ & $-0.39 \pm 0.07$ & - & - & - \\
\hline 71 & 133718.7 & +242303 & IRAS $1334+24$ & 0.108 & $2.50 \pm 0.09$ & $-0.66 \pm 0.03$ & $1.04 \pm 0.02$ & $-0.61 \pm 0.09$ & 0.485 & $0.319 \pm 0.008$ & 1.644 \\
\hline 72 & 134356.7 & +253848 & Ton 730 & 0.087 & $0.55 \pm 0.05$ & $-0.60 \pm 0.05$ & - & - & - & - & - \\
\hline 73 & 135516.6 & +561245 & RX J1355.2+5612 & 0.122 & $0.71 \pm 0.06$ & $-0.63 \pm 0.04$ & - & - & 0.137 & $0.122 \pm 0.008$ & 0.634 \\
\hline 74 & 140516.2 & +255534 & PG $1402+25$ & 0.164 & $0.66 \pm 0.05$ & $-0.55 \pm 0.05$ & $0.99 \pm 0.02$ & $-0.56 \pm 0.10$ & 0.140 & $0.337 \pm 0.009$ & 1.590 \\
\hline 75 & 141336.7 & +702950 & RX J1413.6+7029 & 0.107 & $0.76 \pm 0.07$ & $-0.16 \pm 0.06$ & - & - & 0.199 & $0.105 \pm 0.006$ & 0.401 \\
\hline 76 & 141759.5 & +250812 & NGC 5548 & 0.017 & $4.76 \pm 0.11$ & $-0.14 \pm 0.02$ & $7.95 \pm 0.03$ & $-0.19 \pm 0.02$ & 1.270 & $0.763 \pm 0.019$ & 2.857 \\
\hline 79 & 143104.1 & +281714 & Mkn 684 & 0.046 & $0.53 \pm 0.06$ & $-0.24 \pm 0.07$ & - & - & 0.131 & $0.247 \pm 0.010$ & 1.002 \\
\hline 80 & 144207.5 & +352623 & Mkn 478 & 0.077 & $5.31 \pm 0.11$ & $-0.73 \pm 0.01$ & $3.17 \pm 0.05$ & $-0.75 \pm 0.09$ & 0.958 & $0.119 \pm 0.004$ & 0.660 \\
\hline
\end{tabular}




\begin{tabular}{|c|c|c|c|c|c|c|c|c|c|c|c|}
\hline \multirow{2}{*}{ No. } & \multirow[b]{2}{*}{$\begin{array}{l}\alpha_{2000} \\
\mathrm{~h} \mathrm{~m} \mathrm{~s}\end{array}$} & \multirow{2}{*}{$\begin{array}{l}\delta_{2000} \\
\circ^{\prime} \prime \prime\end{array}$} & \multirow{2}{*}{ Name } & \multirow{2}{*}{$z$} & \multicolumn{2}{|c|}{ RASS } & \multicolumn{2}{|c|}{ PSPC pointed } & \multicolumn{3}{|c|}{ HRI pointed } \\
\hline & & & & & $\begin{array}{c}\mathrm{CR} \\
\operatorname{cts~s}^{-1}\end{array}$ & HR1 & $\begin{array}{c}\mathrm{CR} \\
\operatorname{cts~s}^{-1}\end{array}$ & HR1 & $\begin{array}{l}\text { expected } \\
\text { HRI CR }\end{array}$ & $\begin{array}{c}\mathrm{CR} \\
\mathrm{ctss}^{-1}\end{array}$ & $\begin{array}{c}\text { converted } \\
\text { CR }\end{array}$ \\
\hline 81 & 145108.8 & +270927 & PG $1448+273$ & 0.065 & $0.78 \pm 0.06$ & $-0.03 \pm 0.05$ & - & - & - & - & - \\
\hline 82 & 150401.2 & +102616 & Mkn 841 & 0.036 & $0.77 \pm 0.08$ & $-0.18 \pm 0.06$ & $2.20 \pm 0.01$ & $-0.07 \pm 0.02$ & 0.205 & $0.888 \pm 0.019$ & 3.303 \\
\hline 83 & 152907.5 & +561607 & HS $1529+56$ & 0.100 & $0.79 \pm 0.05$ & $-0.46 \pm 0.03$ & - & - & - & - & - \\
\hline 84 & 155909.7 & +350148 & Mkn 493 & 0.032 & $0.55 \pm 0.06$ & $-0.24 \pm 0.07$ & $0.40 \pm 0.01$ & $-0.23 \pm 0.07-$ & - & - & \\
\hline 85 & 161357.2 & +654311 & Mkn 876 & 0.129 & $0.84 \pm 0.05$ & $-0.08 \pm 0.03$ & $0.98 \pm 0.01$ & $-0.04 \pm 0.05$ & - & - & - \\
\hline 86 & 161809.4 & +361958 & RX J1618.1+3619 & 0.034 & $0.87 \pm 0.05$ & $-0.44 \pm 0.03$ & - & - & 0.193 & $0.113 \pm 0.005$ & 0.509 \\
\hline 87 & 161951.3 & +405848 & KUG $1618+40$ & 0.038 & $0.57 \pm 0.06$ & $-0.56 \pm 0.04$ & - & - & - & - & - \\
\hline 88 & 162456.6 & +755456 & RX J1624.9+7554 & 0.065 & $0.44 \pm 0.04$ & $-0.22 \pm 0.04$ & 3 & 3 & - & - & - \\
\hline 89 & 162756.1 & +552232 & PG $1626+554$ & 0.133 & $0.61 \pm 0.05$ & $-0.37 \pm 0.04$ & $1.05 \pm 0.02$ & $-0.49 \pm 0.09$ & - & - & - \\
\hline 90 & 162901.3 & +400800 & EXO $1627+4014$ & 0.272 & $0.84 \pm 0.05$ & $-0.80 \pm 0.02$ & $0.99 \pm 0.01$ & $-0.77 \pm 0.09$ & - & - & - \\
\hline 91 & 170231.1 & +324720 & HS $1702+32$ & 0.164 & $0.58 \pm 0.05$ & $-0.48 \pm 0.04$ & $0.81 \pm 0.01$ & $-0.62 \pm 0.07$ & - & - & - \\
\hline 92 & 213227.9 & +100820 & II Zw 136 & 0.065 & $1.30 \pm 0.07$ & $+0.01 \pm 0.04$ & $1.16 \pm 0.02$ & $+0.08 \pm 0.04$ & 0.393 & $0.265 \pm 0.011$ & 0.874 \\
\hline 93 & 214636.0 & -305141 & RX J2146.6-3051 & 0.075 & $0.77 \pm 0.06$ & $-0.14 \pm 0.06$ & - & - & - & - & - \\
\hline 94 & 220745.0 & -323501 & A 09.25 & 0.063 & $0.98 \pm 0.07$ & $-0.25 \pm 0.06$ & - & - & - & - & - \\
\hline 95 & 220907.6 & -274836 & NGC 7214 & 0.023 & $0.78 \pm 0.08$ & $-0.25 \pm 0.07$ & $2.69 \pm 0.01$ & $-0.40 \pm 0.02$ & 0.198 & $0.133 \pm 0.02$ & 0.525 \\
\hline 96 & 221653.2 & -445157 & RX J2216.8-4451 & 0.136 & $1.39 \pm 0.08$ & $-0.66 \pm 0.03$ & - & - & 0.279 & $0.140 \pm 0.005$ & 0.698 \\
\hline 97 & 221756.6 & -594130 & RX J2217.8-5941 & 0.160 & $0.83 \pm 0.06$ & $-0.67 \pm 0.04$ & - & - & 0.169 & $0.005 \pm 0.002$ & 0.026 \\
\hline 98 & 223040.3 & -394252 & PKS 2227-399 & 0.318 & $0.55 \pm 0.06$ & $-0.10 \pm 0.08$ & $0.61 \pm 0.01$ & $-0.19 \pm 0.08$ & - & - & - \\
\hline 99 & 224237.7 & -384516 & RX J2242.6-3845 & 0.221 & $0.65 \pm 0.06$ & $-0.70 \pm 0.05$ & - & - & 0.117 & $0.060 \pm 0.010$ & 0.333 \\
\hline 100 & 224520.3 & -465212 & RX J2245.2-4652 & 0.201 & $1.60 \pm 0.09$ & $-0.70 \pm 0.04$ & $1.98 \pm 0.02$ & $-0.71 \pm 0.05$ & 0.305 & $0.218 \pm 0.007$ & 1.147 \\
\hline 101 & 224841.2 & -510953 & RX J2248.6-5109 & 0.102 & $2.58 \pm 0.10$ & $-0.61 \pm 0.03$ & $2.41 \pm 0.02$ & $-0.53 \pm 0.05$ & - & - & - \\
\hline 102 & 225739.0 & -365607 & MS 2254-36 & 0.039 & $2.04 \pm 0.11$ & $-0.56 \pm 0.04$ & $2.29 \pm 0.03$ & $-0.61 \pm 0.06$ & - & - & - \\
\hline 103 & 225845.4 & -260914 & RX J2258.7-2609 & 0.076 & $0.66 \pm 0.09$ & $-0.16 \pm 0.12$ & - & - & 0.128 & $0.122 \pm 0.008$ & 0.625 \\
\hline 104 & 230136.2 & -591320 & RX J2301.6-5913 & 0.149 & $0.91 \pm 0.07$ & $-0.12 \pm 0.06$ & - & - & - & - & - \\
\hline 105 & 230152.0 & -550831 & RX J2301.8-5508 & 0.140 & $0.90 \pm 0.07$ & $-0.63 \pm 0.04$ & $0.65 \pm 0.01$ & $-0.67 \pm 0.07$ & - & - & - \\
\hline 106 & 230437.3 & -350113 & RX J2304.6-3501 & 0.042 & $0.61 \pm 0.08$ & $-0.47 \pm 0.09$ & $0.19 \pm 0.01$ & $-0.48 \pm 0.10$ & - & - & - \\
\hline 107 & 231234.8 & -340420 & RX J2312.5-3404 & 0.202 & $0.43 \pm 0.08$ & $-0.20 \pm 0.12$ & - & - & - & - & - \\
\hline 108 & 231749.9 & -442228 & RX J2317.8-4422 & 0.132 & $0.80 \pm 0.08$ & $-0.79 \pm 0.05$ & - & - & 0.142 & $0.072 \pm 0.005$ & 0.410 \\
\hline 109 & 232511.8 & -323635 & RX J2325.2-3236 & 0.216 & $0.58 \pm 0.10$ & $-0.62 \pm 0.11$ & - & - & 0.115 & $0.085 \pm 0.005$ & 0.425 \\
\hline 110 & 232524.2 & -382649 & IRAS 23226-38 & 0.036 & $1.27 \pm 0.12$ & $-0.11 \pm 0.09$ & - & - & - & - & - \\
\hline 111 & 234328.6 & -145530 & MS 23409-1511 & 0.137 & $0.81 \pm 0.07$ & $-0.44 \pm 0.07$ & $0.89 \pm 0.01$ & $-0.46 \pm 0.04$ & - & - & - \\
\hline 112 & 234924.1 & -312603 & RX J2349-3126 & 0.135 & $0.64 \pm 0.06$ & $-0.53 \pm 0.06$ & $0.11 \pm 0.01$ & $-0.21 \pm 0.13$ & - & - & - \\
\hline 113 & 235728.0 & -302740 & AM 2354-304 & 0.033 & $0.56 \pm 0.06$ & $-0.26 \pm 0.07$ & - & - & - & - & - \\
\hline
\end{tabular}

${ }^{1}$ WPVS007: Because of the small number of photons no hardness ratio is given.

${ }^{2}$ IC 3599: Merged event file of ROR 702704 and 702706 .

${ }^{3}$ RX J1624+75: Only upper limits are available (Grupe et al. :1999b). 


\begin{tabular}{|c|c|c|c|c|c|c|c|c|c|c|c|c|}
\hline \multirow{2}{*}{ No. } & \multirow{2}{*}{ name } & \multicolumn{3}{|c|}{ RASS } & \multicolumn{4}{|c|}{ PSPC-pointed } & \multicolumn{4}{|c|}{ HRI pointed } \\
\hline & & $\begin{array}{c}\text { begin } \\
\text { yymmdd.d }\end{array}$ & $\begin{array}{c}\text { end } \\
\text { yymmdd.d }\end{array}$ & $\begin{array}{r}T_{\mathrm{obs}} \\
\mathrm{s}\end{array}$ & $\begin{array}{c}\text { begin } \\
\text { yymmdd.d }\end{array}$ & $\begin{array}{c}\text { end } \\
\text { yymmdd.d }\end{array}$ & $\begin{array}{r}T_{\mathrm{obs}} \\
\mathrm{s}\end{array}$ & ROR & $\begin{array}{c}\text { begin } \\
\text { yymmdd.d }\end{array}$ & $\begin{array}{c}\text { end } \\
\text { yymmdd.d }\end{array}$ & $\begin{array}{r}T_{\mathrm{obs}} \\
\mathrm{s}\end{array}$ & ROR \\
\hline 1 & Mkn 335 & 900713.8 & 900715.2 & 375 & 910629.8 & 910630.8 & 24337 & 700101 & 971220.4 & 971221.3 & 11014 & 601123 \\
\hline 2 & ESO $242-G 8$ & 901125.4 & 901127.3 & 238 & 930604.4 & 930605.8 & 6208 & 701148 & 951225.5 & 951225.5 & 2291 & 702639 \\
\hline 3 & WPVS 007 & 901123.2 & 901125.4 & 299 & 931111.5 & 931113.5 & 9691 & 701527 & 960613.4 & 960624.7 & 19863 & 702705 \\
\hline 4 & RX J0057-22 & 901216.9 & 901218.5 & 466 & 920618.6 & 920618.7 & 2961 & 701141 & - & - & - & - \\
\hline 5 & QSO 0056-36 & 901209.4 & 901211.5 & 528 & - & - & - & - & 961221.0 & 961230.0 & 2256 & 702905 \\
\hline 6 & RX J0100-51 & 901128.0 & 901130.2 & 306 & - & - & - & - & 960602.1 & 960625.4 & 5155 & 702633 \\
\hline 7 & RX J0105-14 & 900712.9 & 900714.8 & 579 & - & - & - & - & - & - & - & - \\
\hline 8 & RX J0117-38 & 901212.0 & 901214.4 & 540 & - & - & - & - & - & - & - & - \\
\hline 9 & MS 0117-28 & 901218.8 & 901220.9 & 412 & 911228.5 & 911229.3 & 4467 & 700445 & - & - & - & - \\
\hline 10 & RX J0128-18 & 900715.9 & 910117.0 & 418 & - & - & - & - & - & - & - & - \\
\hline 11 & IRAS01267-21 & 900715.2 & 910116.2 & 344 & - & - & - & - & 960112.4 & 960112.5 & 4853 & 702655 \\
\hline 12 & RX J0148-27 & 900715.9 & 910117.2 & 504 & 920609.4 & 920610.1 & 6682 & 701185 & - & - & - & - \\
\hline 13 & RX J0152-23 & 901229.5 & 901231.2 & 608 & - & - & - & - & 960101.8 & 960112.3 & 4978 & 702654 \\
\hline 14 & Mkn 1044 & 910114.3 & 910119.3 & 370 & 920809.5 & 920810.5 & 2836 & 700792 & - & - & - & - \\
\hline 15 & Mkn 1048 & 910119.0 & 910120.4 & 382 & 930809.2 & 930810.0 & 6191 & 701599 & 960115.8 & 960115.0 & 2411 & 600866 \\
\hline 16 & RX J0311-20 & 910122.9 & 910124.3 & 319 & - & - & - & - & - & - & - & - \\
\hline 17 & RX J0319-26 & 910122.9 & 910124.3 & 263 & 920818.8 & 920821.3 & 6823 & 701052 & - & - & - & - \\
\hline 18 & RX J0323-49 & 910102.4 & 910105.7 & 745 & 921222.9 & 921223.9 & 5914 & 701183 & 960817.7 & 960819.7 & 2428 & 702670 \\
\hline 19 & ESO 301-G13 & 910111.4 & 910114.7 & 657 & - & - & - & - & 960102.7 & 960105.0 & 3805 & 702657 \\
\hline 20 & VCV0331-37 & 910121.0 & 910123.1 & 244 & - & - & - & - & 970713.3 & 970713.4 & 3427 & 702902 \\
\hline 21 & RX J0349-47 & 910113.7 & 910120.2 & 421 & - & - & - & - & 960117.4 & 960117.5 & 2049 & 600869 \\
\hline 22 & Fair 1116 & 910124.0 & 910125.2 & 167 & - & - & - & - & 970712.6 & 970712.6 & 2754 & 702903 \\
\hline 23 & CTS 44 & 910805.4 & 910810.8 & 588 & - & - & - & - & - & - & - & - \\
\hline 24 & RX J0412-47 & 910124.0 & 910125.2 & 163 & - & - & - & - & 951230.0 & 951230.2 & 5367 & 702662 \\
\hline 25 & RX J0426-57 & 910109.1 & 910119.3 & 796 & 920407.3 & 920407.8 & 4094 & 700034 & 960816.5 & 960816.6 & 4703 & 702038 \\
\hline 26 & Fair 303 & 910122.1 & 910125.2 & 290 & 911220.4 & 911220.5 & 2669 & 800043 & - & - & - & - \\
\hline 27 & RX J0437-47 & 900730.6 & 910812.4 & 1037 & 920920.7 & 920921.8 & 6142 & 701184 & 970922.1 & 970926.1 & 23114 & 400897 \\
\hline 28 & RX J0439-53 & 910805.3 & 910809.8 & 148 & 970220.6 & 970220.7 & 1370 & 190537 & - & - & - & - \\
\hline 29 & Н 0439-272 & 900812.6 & 900814.6 & 122 & 910310.2 & 910310.3 & 4955 & 700035 & - & - & - & - \\
\hline 30 & 1 ES 0614-58 & 900920.7 & 901003.2 & 2792 & - & - & - & - & - & - & - & - \\
\hline 31 & RX J0859+48 & 901014.9 & 901017.0 & 448 & - & - & - & - & - & - & - & - \\
\hline 32 & RX J0902-07 & 901102.5 & 901104.2 & 410 & - & - & - & - & - & - & - & - \\
\hline 33 & Mkn 110 & 901018.2 & 901020.0 & 617 & 911101.8 & 911102.8 & 6013 & 700262 & 951016.8 & 951101.9 & 30308 & 702627 \\
\hline 34 & PG $0953+41$ & 901029.4 & 901031.3 & 485 & 920430.2 & 920430.4 & 7014 & 700526 & - & - & - & - \\
\hline 35 & RX J1005+43 & 901030.1 & 901101.0 & 686 & - & - & - & - & 951101.5 & 951108.4 & 4711 & 702656 \\
\hline 36 & RX J1007+22 & 901108.4 & 901110.1 & 599 & - & - & - & - & - & - & - & - \\
\hline 37 & CBS 126 & 901104.1 & 901105.9 & 642 & - & - & - & - & 951030.8 & 951031.1 & 3901 & 702651 \\
\hline 38 & HS $1019+37$ & 901104.4 & 901106.3 & 643 & 931029.7 & 931030.2 & 13510 & 800491 & - & - & - & - \\
\hline 39 & Mkn 141 & 901019.2 & 901021.9 & 694 & - & - & - & - & 961010.4 & 961010.8 & 3589 & 702664 \\
\hline 40 & Mkn 142 & 901029.3 & 901031.5 & 762 & 931021.2 & 931022.6 & 8427 & 600624 & - & - & - & - \\
\hline
\end{tabular}




\begin{tabular}{|c|c|c|c|c|c|c|c|c|c|c|c|c|}
\hline \multirow{2}{*}{ No. } & \multirow{2}{*}{ name } & \multicolumn{3}{|c|}{ RASS } & \multicolumn{4}{|c|}{ PSPC-pointed } & \multicolumn{4}{|c|}{ HRI pointed } \\
\hline & & $\begin{array}{c}\text { begin } \\
\text { yymmdd.d }\end{array}$ & $\begin{array}{c}\text { end } \\
\text { yymmdd.d }\end{array}$ & $\begin{array}{r}T_{\mathrm{obs}} \\
\mathrm{s}\end{array}$ & $\begin{array}{c}\text { begin } \\
\text { yymmdd.d }\end{array}$ & $\begin{array}{c}\text { end } \\
\text { yymmdd.d }\end{array}$ & $\begin{array}{r}T_{\mathrm{obs}} \\
\mathrm{s}\end{array}$ & ROR & $\begin{array}{c}\text { begin } \\
\text { yymmdd.d }\end{array}$ & $\begin{array}{c}\text { end } \\
\text { yymmdd.d }\end{array}$ & $\begin{array}{r}T_{\mathrm{obs}} \\
\mathrm{s}\end{array}$ & ROR \\
\hline 41 & RX J1034+39 & 901106.8 & 901108.8 & 525 & 911118.8 & 911119.1 & 4617 & 700551 & - & - & - & - \\
\hline 42 & RX J1117+65 & 901025.8 & 901028.8 & 874 & - & - & - & - & 961022.4 & 961022.4 & 2169 & 702892 \\
\hline 43 & PG $1115+407$ & 901115.2 & 901117.3 & 446 & 930520.4 & 930520.8 & 6489 & 700801 & 951114.9 & 951115.0 & 3645 & 702191 \\
\hline 44 & Ton 1388 & 901124.8 & 901126.5 & 422 & 910529.3 & 910530.2 & 24294 & 700228 & 950528.0 & 950528.1 & 1774 & 701690 \\
\hline 45 & EXO $1128+69$ & 901023.1 & 901026.3 & 1099 & - & - & - & - & - & - & - & - \\
\hline 46 & B2 $1128+31$ & 901122.8 & 901124.4 & 423 & - & - & - & - & - & - & - & - \\
\hline 47 & SBS $1136+579$ & 901106.2 & 901108.9 & 491 & 930530.1 & 930530.3 & 5018 & 800402 & - & - & - & - \\
\hline 48 & Z 1136+3412 & 901123.2 & 901125.1 & 422 & 930518.5 & 930519.1 & 2046 & 201120 & - & - & - & - \\
\hline 49 & Was 26 & 901129.5 & 901201.3 & 602 & 921207.0 & 921207.5 & 4889 & 701149 & - & - & - & - \\
\hline 50 & CASG 855 & 901122.8 & 901124.3 & 378 & - & - & - & - & - & - & - & - \\
\hline 51 & Mkn 1310 & 901214.8 & 901216.5 & 481 & 920707.8 & 920707.9 & 3341 & 201367 & - & - & - & - \\
\hline 52 & NGC 4051 & 901121.8 & 901123.9 & 506 & 911116.0 & 911116.9 & 28459 & 700557 & 950520.5 & 950520.6 & 2587 & 701884 \\
\hline 53 & GQ Com & 901201.9 & 901203.8 & 666 & 910604.2 & 910605.2 & 25991 & 700232 & 960621.4 & 960621.5 & 4639 & 702171 \\
\hline 54 & RX J1209+32 & 901130.7 & 901202.7 & 210 & 930523.5 & 930523.8 & 2748 & 701051 & - & - & - & - \\
\hline 55 & PG $1211+143$ & 901210.5 & 901212.3 & 609 & 911217.1 & 911224.7 & 3803 & 700018 & - & - & - & - \\
\hline 56 & Mkn 766 & 901203.9 & 901205.9 & 525 & 921208.6 & 921210.2 & 6774 & 701091 & 960621.7 & 960621.7 & 1518 & 702633 \\
\hline 57 & $3 \mathrm{C} 273$ & 901218.9 & 901220.7 & 496 & 911214.3 & 911215.2 & 6140 & 700191 & 941222.1 & 941222.1 & 2088 & 701961 \\
\hline 58 & RX J1231+70 & 901026.4 & 901030.2 & 1005 & - & - & - & - & 960504.9 & 960505.7 & 4285 & 702652 \\
\hline 59 & Mkn 771 & 901211.9 & 901213.7 & 604 & 911213.3 & 911228.2 & 6402 & 700435 & - & - & - & - \\
\hline 60 & CBS 150 & 901206.7 & 901208.8 & 690 & 920702.3 & 920702.7 & 3120 & 701050 & - & - & - & - \\
\hline 61 & MCG+08-23-06 & 901127.7 & 901130.1 & 661 & - & - & - & - & - & - & - & - \\
\hline 62 & IC 3599 & 901209.9 & 901211.9 & 658 & 920630.3 & 920701.9 & 5422 & 701097 & 960630.5 & 960702.1 & 31621 & 1 \\
\hline 63 & NGC 4593 & 900715.1 & 910115.8 & 180 & 920714.3 & 920714.3 & 1261 & 701012 & 960114.9 & 960116.4 & 1912 & 701885 \\
\hline 64 & IRAS $1239+33$ & 901207.3 & 901209.3 & 707 & 911215.7 & 920104.2 & 19536 & 600129 & - & - & - & - \\
\hline 65 & PG $1244+026$ & 900714.1 & 900715.3 & 239 & 911222.2 & 911224.8 & 5486 & 700020 & - & - & - & - \\
\hline 66 & RX J1304+02 & 901227.9 & 910117.2 & 401 & - & - & - & - & - & - & - & - \\
\hline 67 & PG $1307+085$ & 900716.2 & 910117.0 & 509 & 920713.5 & 920719.0 & 7682 & 700229 & 950714.7 & 950714.9 & 2950 & 400625 \\
\hline 68 & RX J1314+34 & 901213.9 & 901216.1 & 740 & 920706.8 & 920706.8 & 2526 & 700802 & 960108.8 & 960110.1 & 5916 & 702660 \\
\hline 69 & RX J1319+52 & 901129.8 & 901202.8 & 714 & - & - & - & - & - & - & - & - \\
\hline 70 & PG $1322+659$ & 901110.9 & 901114.5 & 810 & 921130.0 & 921201.9 & 8387 & 700803 & - & - & - & - \\
\hline 71 & IRAS $1334+24$ & 910115.3 & 910116.8 & 506 & 920107.7 & 920114.6 & 3281 & 700553 & 960716.7 & 960721.8 & 6207 & 702625 \\
\hline 72 & Ton 730 & 900716.3 & 910117.1 & 592 & - & - & - & - & - & - & - & - \\
\hline 73 & RX J1355+56 & 901202.1 & 901205.6 & 829 & - & - & - & - & 970529.8 & 970529.9 & 2577 & 702897 \\
\hline 74 & PG $1402+25$ & 901231.4 & 910102.6 & 627 & 920107.7 & 920113.3 & 2692 & 700226 & 960114.2 & 960114.3 & 4379 & 702152 \\
\hline 75 & RX J1413+70 & 901104.2 & 901109.2 & 501 & - & - & - & - & 951023.9 & 951114.3 & 4140 & 702659 \\
\hline 76 & NGC 5548 & 910104.1 & 910117.5 & 631 & 921225.4 & 921225.7 & 8126 & 700536 & 950124.6 & 950125.1 & 2260 & 701950 \\
\hline 77 & Q 1421-0013 & 910119.5 & 910120.8 & 442 & - & - & - & - & - & - & - & - \\
\hline 78 & Mkn 813 & 910109.2 & 910111.1 & 684 & - & - & - & - & - & - & - & - \\
\hline 79 & Mkn 684 & 910105.8 & 910118.7 & 538 & - & - & - & - & 970719.9 & 970720.1 & 2835 & 702907 \\
\hline 80 & Mkn 478 & 910104.2 & 910118.0 & 692 & 920117.6 & 920117.8 & 2354 & 700559 & 971224.1 & 971224.5 & 9632 & 704273 \\
\hline
\end{tabular}




\begin{tabular}{|c|c|c|c|c|c|c|c|c|c|c|c|c|}
\hline \multirow{2}{*}{ No. } & \multirow{2}{*}{ name } & \multicolumn{3}{|c|}{ RASS } & \multicolumn{4}{|c|}{ PSPC-pointed } & \multicolumn{4}{|c|}{ HRI pointed } \\
\hline & & $\begin{array}{c}\text { begin } \\
\text { yymmdd.d }\end{array}$ & $\begin{array}{c}\text { end } \\
\text { yymmdd.d }\end{array}$ & $\begin{array}{r}T_{\text {obs }} \\
\mathrm{s}\end{array}$ & $\begin{array}{c}\text { begin } \\
\text { yymmdd.d }\end{array}$ & $\begin{array}{c}\text { end } \\
\text { yymmdd.d }\end{array}$ & $\begin{array}{r}T_{\mathrm{obs}} \\
\mathrm{s}\end{array}$ & ROR & $\begin{array}{c}\text { begin } \\
\text { yymmdd.d }\end{array}$ & $\begin{array}{c}\text { end } \\
\text { yymmdd.d }\end{array}$ & $\begin{array}{r}T_{\mathrm{obs}} \\
\mathrm{s}\end{array}$ & ROR \\
\hline 81 & PG $1448+273$ & 910111.8 & 910114.1 & 701 & - & - & - & - & - & - & - & - \\
\hline 82 & Mkn 841 & 910124.4 & 910125.8 & 451 & 920120.2 & 920126.1 & 16842 & 700257 & 950801.0 & 950808.9 & 2699 & 701677 \\
\hline 83 & HS $1529+56$ & 900714.2 & 910117.1 & 1287 & - & - & - & - & - & - & - & - \\
\hline 84 & Mkn 493 & 910807.3 & 910810.0 & 553 & 920201.4 & 920129.6 & 8038 & 700096 & - & - & - & - \\
\hline 85 & Mkn 876 & 901201.6 & 901210.8 & 1906 & 911123.3 & 911203.9 & 6514 & 700230 & - & - & - & - \\
\hline 86 & RX J1618+36 & 900730.6 & 910803.1 & 1142 & - & - & - & - & 960220.9 & 960222.8 & 4473 & 702658 \\
\hline 87 & KUG $1618+40$ & 900730.3 & 910812.5 & 1046 & - & - & - & - & - & - & - & - \\
\hline 88 & RX J1624+75 & 901007.4 & 901015.9 & 1766 & 920112.9 & 920113.8 & 2923 & 141829 & - & - & - & - \\
\hline 89 & PG $1626+554$ & 910114.5 & 910123.2 & 1122 & 930803.5 & 930804.7 & 2328 & 701372 & - & - & - & - \\
\hline 90 & EXO $1627+401$ & 900801.2 & 900804.9 & 1188 & 930730.2 & 930730.8 & 5175 & 701507 & - & - & - & - \\
\hline 91 & HS $1702+32$ & 900818.9 & 910218.1 & 1117 & 930803.3 & 930803.7 & 11317 & 800530 & - & - & - & - \\
\hline 92 & II Zw 136 & 901111.5 & 901113.3 & 562 & 930529.8 & 930605.1 & 8622 & 701252 & 951117.2 & 951117.2 & 2155 & 702638 \\
\hline 93 & RX J2146-30 & 901031.5 & 901102.3 & 475 & - & - & - & - & - & - & - & - \\
\hline 94 & A 09.25 & 901104.4 & 901106.1 & 342 & - & - & - & - & - & - & - & - \\
\hline 95 & NGC 7214 & 901106.6 & 901108.4 & 328 & 920501.1 & 920502.2 & 15756 & 600175 & 951113.0 & 951114.8 & 34040 & 800848 \\
\hline 96 & RX J2216-44 & 901031.6 & 901102.6 & 471 & - & - & - & - & 951108.4 & 951108.4 & 57 & 702650 \\
\hline 97 & RX J2217-59 & 901022.5 & 901024.8 & 587 & - & - & - & - & 980415.7 & 980418.0 & 9760 & 601124 \\
\hline 98 & PKS 2227-399 & 901106.1 & 901107.6 & 425 & 930530.7 & 930530.8 & 3652 & 701081 & - & - & - & - \\
\hline 99 & RX J2242-38 & 901109.1 & 901110.7 & 472 & - & - & - & - & 961201.8 & 961204.4 & 756 & 702895 \\
\hline 100 & RX J2245-46 & 901105.2 & 901107.1 & 340 & 921118.9 & 921128.6 & 5507 & 701186 & 941129.4 & 941130.8 & 4515 & 201870 \\
\hline 101 & RX J2248-51 & 901103.1 & 901104.7 & 499 & 931102.3 & 931103.0 & 4517 & 701601 & - & - & - & - \\
\hline 102 & MS 2254-36 & 901113.0 & 901114.3 & 284 & 920502.8 & 920502.9 & 5514 & 700554 & - & - & - & - \\
\hline 103 & RX J2258-26 & 901118.1 & 901120.0 & 143 & - & - & - & - & 961117.5 & 961118.4 & 2538 & 702909 \\
\hline 104 & RX J2301-59 & 901029.8 & 901101.3 & 485 & - & - & - & - & - & - & - & - \\
\hline 105 & RX J2301-55 & 901102.3 & 901104.7 & 541 & 930518.7 & 930531.5 & 8806 & 701189 & - & - & - & - \\
\hline 106 & RX J2304-35 & 901115.3 & 901117.2 & 204 & 921201.7 & 921204.3 & 9101 & 701187 & - & - & - & - \\
\hline 107 & RX J2312-34 & 901117.9 & 901119.0 & 175 & - & - & - & - & - & - & - & - \\
\hline 108 & RX J2317-44 & 901113.0 & 901114.3 & 269 & - & - & - & - & 970507.3 & 970507.4 & 3050 & $702889^{2}$ \\
\hline 109 & RX J2325-32 & 901121.1 & 901122.1 & 104 & - & - & - & - & 961130.7 & 961130.8 & 3953 & 702896 \\
\hline 110 & IRAS 23226-3 & 901118.0 & 901119.2 & 142 & - & - & - & - & - & - & - & - \\
\hline 111 & MS 23409-151 & 901203.1 & 901204.7 & 341 & 921220.7 & 921221.6 & 14428 & 701205 & - & - & - & - \\
\hline 112 & RX J2349-31 & 901126.7 & 901128.7 & 452 & 930531.1 & 930531.3 & 8148 & 701190 & - & - & 一 & - \\
\hline 113 & AM 2354-304 & 901129.1 & 901130.6 & 446 & - & - & - & - & - & - & - & - \\
\hline
\end{tabular}

${ }^{1}$ IC 3599: Merged event file of ROR 702704 and 702706

${ }^{2}$ RX J2317-44: ROR = 702889h-1, 702889 is empty. 
Figure 1 shows that most sources show variability on long timescales of years by factors of about 2-3 (see also Table 1), but do not show dramatic changes in their count rates. However four sources are highly variable in X-rays. Three of these sources are considered to be X-ray transient AGN: RX J1624.9+7554 (Grupe et al. 1999b), WPVS007 (Grupe et al. 1995b), and IC 3599 (Brandt et al. 1995; Grupe et al. 1995a). The other source, RX J2217.9-5941, has changed its count rate between RASS and pointed observations by a factor of more than 30 (Grupe et al. 2001, and see below). We can exclude line-of-sight Cataclysmic Variables in our Galaxy as the origin of the transience (see discussions in the separate papers of the sources). Four sources have become brighter in their pointed PSPC observation by a factor of more than 2 . The sources with the highest count rate ratios are Mkn 771, Mkn 841, NGC 7214, and Mkn 110.

We performed two tests for variability, a $\chi^{2}$ test for the RASS data (short term behavior) and a check on the amplitude change per unit time for the long term behavior. Figure 2 displays the results of variability tests as a function of the X-ray luminosity in the rest-frame energy $0.2-2.0 \mathrm{keV}$. In the left panel of Fig. 2 the short term variability of the RASS data is displayed (NGC 4051 and Mkn 766 are off the plot). For objects which have RASS observations that were about half a year apart (see Table 3), only the one with the longest exposure was used. A RASS coverage was typically a few days (see Table 2 for details) with a time increment of $96 \mathrm{~min}$ (the ROSAT orbit). The reduced $\chi^{2}$ was calculated from the mean count rate taking the count rate errors into account. We see that there is a slight dependence of the variability strength on luminosity. Low-luminosity sources tend to be slightly more variable than high-luminosity sources. However, Mkn 478 and RX J1304+02, and RX J2217-59 fall off this trend. The two sources with the strongest variability on short timescales are NGC 4051 and Mkn 766, both well-known for their strong variability (see e.g. Peterson et al. 2000; Leighly et al. 1996). The other two sources with relatively strong variability, RX J1304+02 and RX J2217-59 are "new". On long timescales, RX J1304+02 lacks in further pointed observations and RX J2217-59 is a transient candidate (Grupe et al. 2001 and see below). The X-ray transient AGN do not show unusually strong variability on short timescales.

The long-term behaviour is shown in the right panel of Fig. 2. We also see a slight tendency for low-luminosity sources to be more variable. We took all sources into account for which at least two observations existed that were more than half a year apart. This includes also those sources for which two RASS coverages existed (see Table 3, marked as triangles in Fig. 2). Clearly, in the longterm behaviour, the transients have the highest change in amplitude over time. Two other sources, NGC 4593 and HS1702+32 also show very strong variability on long-timescales. Note that our transient candidate RX J2217.9-5941 is the source with the strongest variability between RASS and HRI observation.

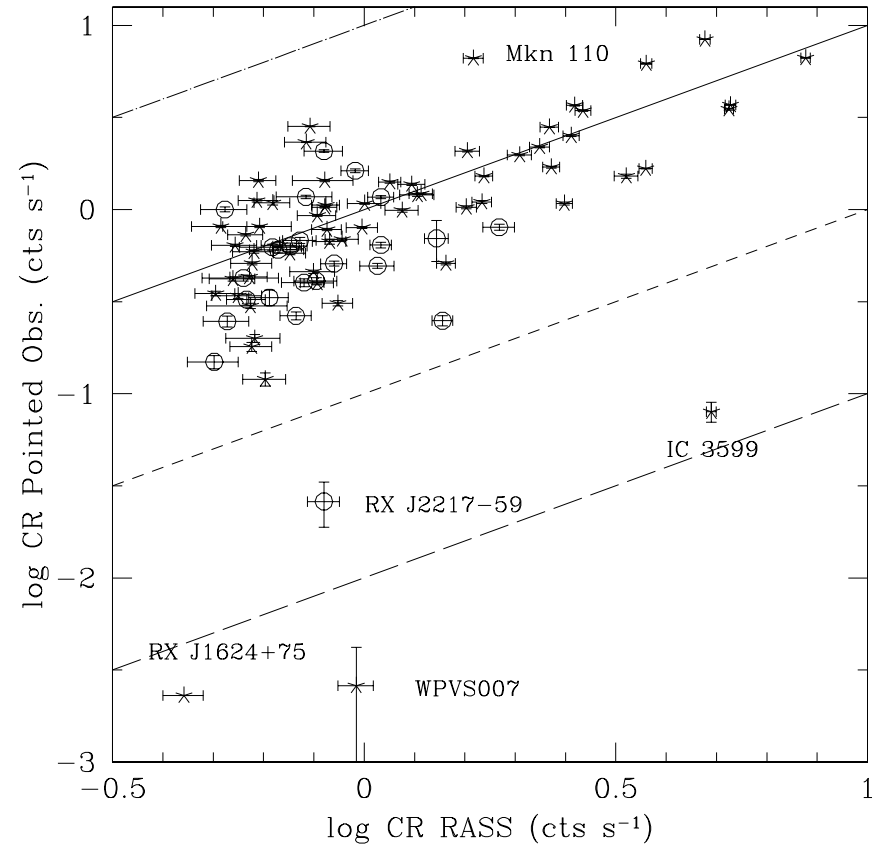

Fig. 1. RASS vs. pointed observation count rate. PSPC observations are marked as stars and HRI observations as open circles. The solid line represents no change between RASS and pointed observation, the dot-dashed line represents to a factor of 10 , the short-dashed line to a factor of 0.1 , and the long-dashed line to a factor of 0.01 . The HRI count rates were converted into PSPC count rates (see Sect. 2)

Figure 3 displays the variability strength $\chi^{2} / \nu$ (same as in Fig. 2) as a function of the X-ray spectral slope $\alpha_{\mathrm{x}}$. We found that sources with steep X-ray spectra tend to show stronger variabilities. The dashed lines in Fig. 3 mark the medians of $\alpha_{\mathrm{x}}$ and $\chi^{2} / \nu$ without the high variability sources NGC 4051 and Mkn 766 and the source with the steepest X-ray spectrum, WPVS007. Dividing our sample in a steep and flat X-ray spectrum (divided by the median $\alpha_{\mathrm{x}}=1.67$ ) we find a mean $\chi^{2} / \nu=1.50$ (with a median of 1.48) for the flat X-ray slope sub-sample and $\chi^{2} / \nu=2.00$ (median 1.72) for the step X-ray spectrum objects.

\subsection{Spectral variability}

Figure 4 shows the hardness ratios of the RASS vs. the pointed PSPC observations. Most sources do not show dramatic spectral changes. Only two objects, RX J2349.3-3126 (see below) and 3C 273, may have varied. Indeed, the difference between the hardness ratios of the RASS and the pointed PSPC observations is small: the mean hardness ratio increased by 0.03 . Figure 5 displays the count rate ratio vs. the difference in the hardness ratios between the RASS and the later pointed PSPC observations. The purpose of this plot is to demonstrate how the sources have changed their spectra with count rate. Obviously, there is no clear correlation between both quantities, the changes going in all possible directions. Spectral changes can also be studied, of course, by using the spectral index $\alpha_{\mathrm{x}}$. However, the $\alpha_{\mathrm{x}}$ depend on model 
Table 3. List of sources that were observed twice during the RASS half a year apart. Observing dates give the beginning of the RASS coverage in yymmdd, the exposure time $T_{\exp }$ is given in $\mathrm{s}$, and the count rate in $\mathrm{cts} \mathrm{s}^{-1}$

\begin{tabular}{llrrrrrc}
\hline \multirow{2}{*}{$\#$ name } & \multicolumn{3}{c}{ first observation } & \multicolumn{3}{c}{ second observation } \\
& & UT date & $T_{\exp }$ & CR & UT date & $T_{\exp }$ & CR \\
\hline 10 & RX J0128-18 & 900715 & 99.4 & $0.088 \pm 0.130$ & 910115 & 318.5 & $1.056 \pm 0.434$ \\
11 & IRAS 01267-21 & 900715 & 210.0 & $0.724 \pm 0.259$ & 910115 & 133.8 & $0.837 \pm 0.409$ \\
12 & RX J0148-27 & 900715 & 89.4 & $1.760 \pm 0.356$ & 901227 & 414.1 & $2.515 \pm 0.538$ \\
63 & NGC 4593 & 900715 & 50.1 & $1.440 \pm 0.663$ & 910116 & 130.2 & $3.908 \pm 0.373$ \\
67 & PG 1307+085 & 900716 & 66.0 & $0.506 \pm 0.264$ & 910115 & 443.1 & $0.652 \pm 0.261$ \\
83 & HS 1529+56 & 900714 & 583.6 & $0.963 \pm 0.293$ & 901227 & 703.7 & $0.639 \pm 0.261$ \\
87 & KUG 1618+40 & 900730 & 473.9 & $0.527 \pm 0.247$ & 910810 & 572.2 & $0.602 \pm 0.331$ \\
91 & HS 1702+32 & 900818 & 810.6 & $0.345 \pm 0.201$ & 910217 & 306.7 & $1.130 \pm 0.406$ \\
\hline
\end{tabular}
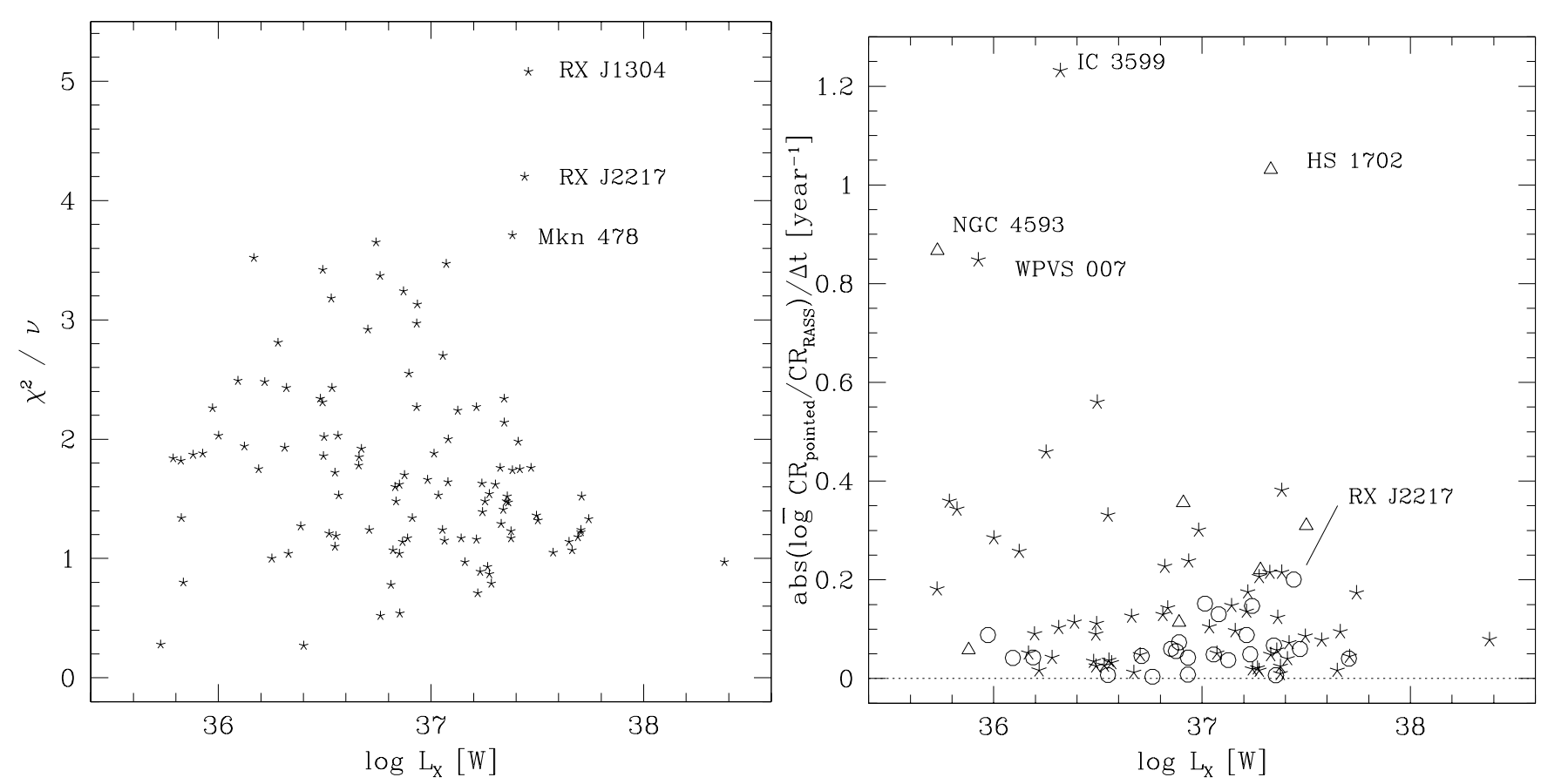

Fig. 2. Variability checks for the soft X-ray AGN sample; the left panel shows the short-term variability in the RASS data and the right panel the long-term variability for all objects for which more than one observation exists (see text). (RASS vs. PSPC observations are marked as stars, RASS vs. HRI as open circles, and RASS vs. RASS as open triangles)

fitting. We prefer to compare hardness ratios because they are determined directly from the observed counts, and the uncertainties are more simply interpreted. The mean change in hardness ratio for small count rate changes (ratios between 0.8 and 1.2 ) turns out to be $0.04 \pm 0.01$. This might be due to some small losses of hard photons by the off-axis correction for the RASS data as compared to the pointing data, which are usually taken on-axis, and therefore not be an intrinsic property of our sample.

\subsection{Spectral analysis}

Table 4 lists the spectral analysis of the RASS and pointed PSPC data. For the power-law fits to the RASS and pointed PSPC data with the absorption parameter $N_{\mathrm{H}}$ fixed to the Galactic value, the X-ray fluxes are given in the restframe ROSAT energy range between $0.2-2.0 \mathrm{keV}$.
The mean X-ray slope of the RASS observation using a power-law fit with fixed $N_{\mathrm{H}}$ is $\alpha_{\mathrm{x}}=1.81$ with a median of 1.67. This is slightly flatter than we found for our original sample ( $\left\langle\alpha_{\mathrm{x}}\right\rangle=2.10$; Grupe et al. 1998a). The mean $\mathrm{X}$-ray luminosity derived from this spectral model is log $L_{\mathrm{X}}=36.87[\mathrm{~W}]$ with a median of 36.91 . The object with the highest luminosity is $3 \mathrm{C} 273\left(\log L_{\mathrm{X}}=38.4\right)$. Dividing the sample into low and high luminosity subsamples using the median of $\log L_{\mathrm{X}}$ as the borderline, we find a difference in the distribution of the X-ray slope $\alpha_{\mathrm{x}}$. The lowluminosity sub-sample has a mean of $\left\langle\alpha_{\mathrm{x}}\right\rangle=1.61$ (median=1.54), the high-luminosity sub-sample $\left\langle\alpha_{\mathrm{x}}\right\rangle=1.89$ $($ median $=1.92)$.

\subsection{Individual $X$-ray transient $A G N$}

Here, we present new results of HRI observations of the X-ray transient AGN IC3599 and WPVS007. We briefly 


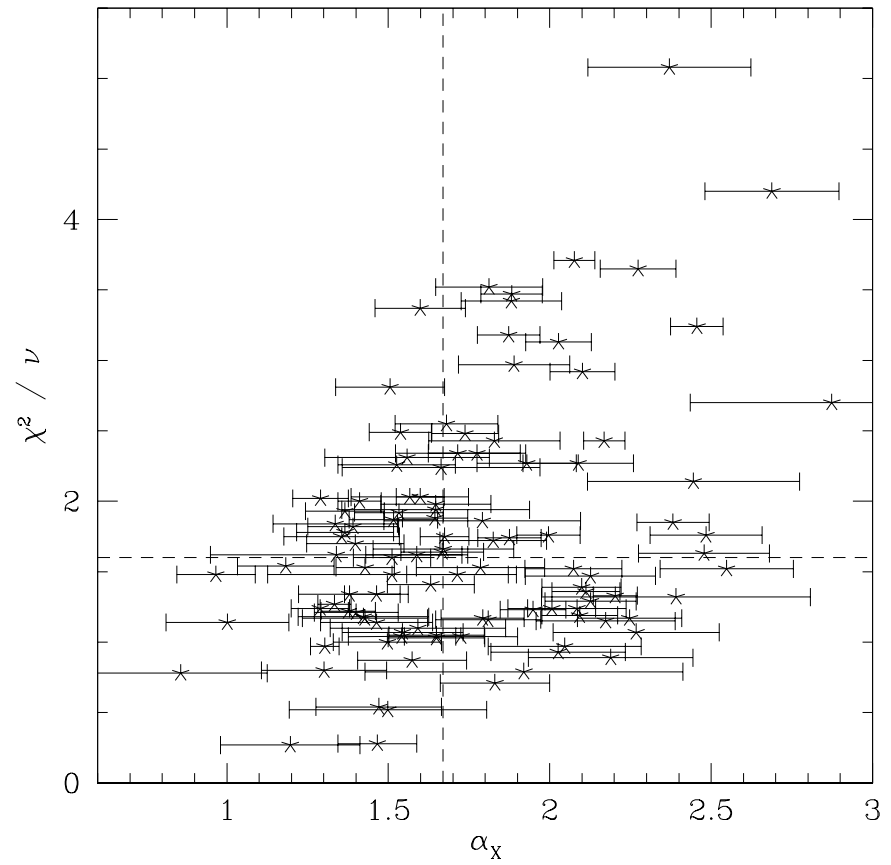

Fig. 3. Short-term $\chi^{2}$ variability vs. X-ray spectral slope $\alpha_{\mathrm{x}}$. The dashed lines mark the medians in $\alpha_{\mathrm{x}}$ and $\chi^{2} / \nu$. Three objects are off the plot, NGC 4051, Mkn 766 and WPVS007 (with positions at $1.62,18.54 ; 1.11,8.86$; and $8.70,1.88$ respectively)

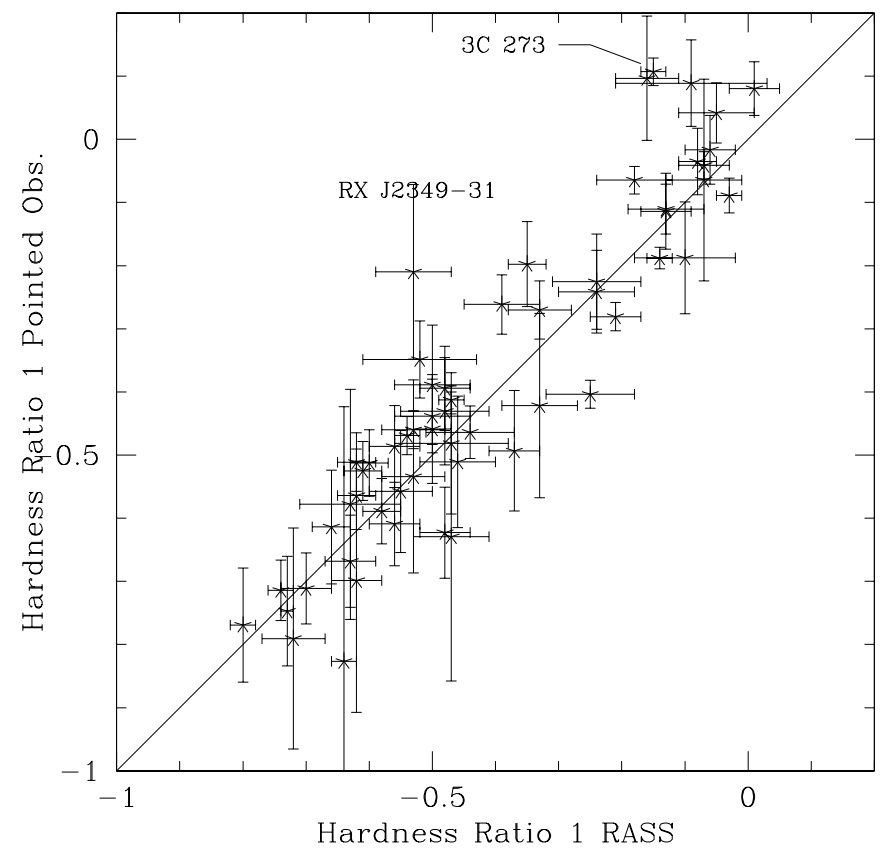

Fig. 4. RASS vs. pointed observation hardness ratio

review the other most recently discovered transients, $\mathrm{RX}$ J1624.9+7554 and RX J2217.9-5941.

\subsubsection{IC 3599}

The Seyfert 2 galaxy IC 3599 was one of the brightest AGN of our sample during the RASS (4.90 PSPC $\mathrm{cts} \mathrm{s}^{-1}$ ) and was seen even with the Wide Field Camera (WFC)

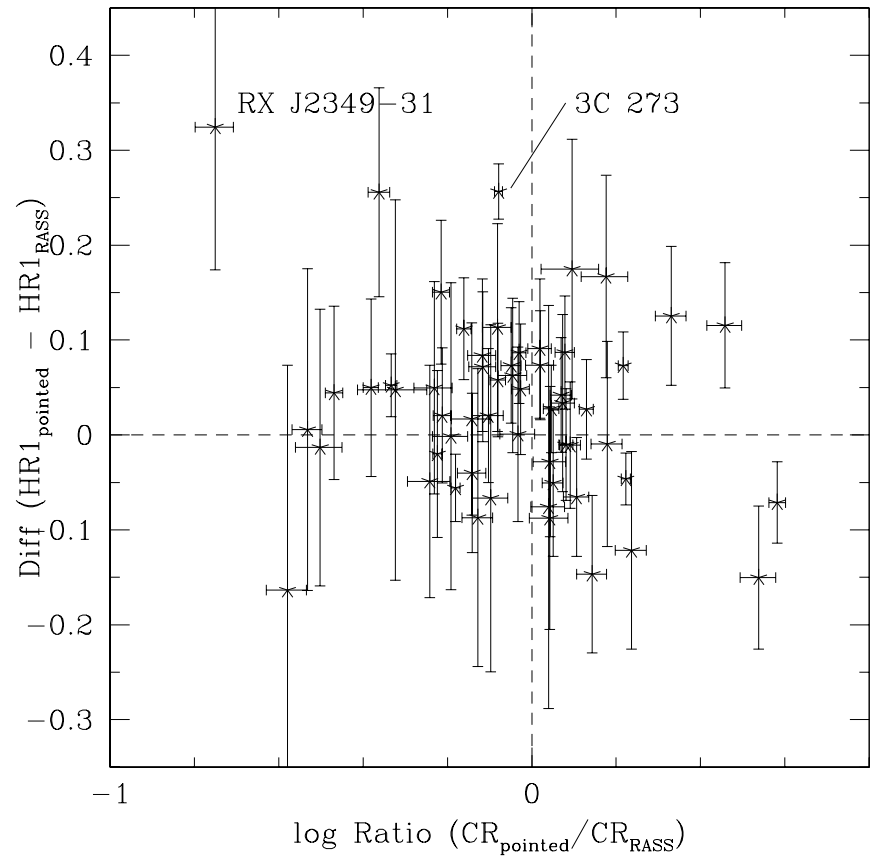

Fig. 5. Ratio of the count rates of pointed PSPC and RASS vs. the difference between their $H R 1$. The transients IC 3599 and WPVS007 fall outside the boundaries of the plot

that was attached to ROSAT (Pounds et al. 1993; Edelson et al. 1999). It became fainter in pointed PSPC observations in the following years by a factor of about 100 (see Grupe et al. 1995a; Brandt et al. 1995). We made two ROSAT HRI observations between 1996-06-30 and 1996-07-02 to monitor its long term behaviour. In order to get better photon statistics on the source, both event files were merged into one. From this merged event file with a total exposure time of $31621 \mathrm{~s}$ an HRI count rate of $4.62 \pm 0.4910^{-3}$ was measured, which corresponds to 0.025 PSPC cts s$^{-1}$ (see Table 1) and an X-ray luminosity in the $0.2-2.0 \mathrm{keV}$ band of $\log L_{\mathrm{X}}=33.6$. Figure 6 displays the long-term light curve. IC 3599 has shown a response in its optical emission line spectrum. Shortly after the RASS, highly ionized iron lines like FeXIV were observed (see Brandt et al. 1995). A year later, these lines disappeared but other lower ionized iron lines like FeVII and FeX appeared (Grupe et al. 1995a).

Monitoring this object in the optical until now shows that these lines are slowly fading. The interpretation of the X-ray outburst in IC 3599 is that it is the result of disk instabilities or tidal disruption of a star by the central back hole (see Brandt et al. 1995; Grupe et al. 1995a). A similar outburst event has been reported in the galaxy NGC 5905 (Komossa \& Bade 1999).

\subsubsection{WPVS007}

The Narrow-line Seyfert 1 galaxy WPVS007 was the softest AGN detected during the RASS (Grupe et al. 1995b) and it was bright (about $1 \mathrm{cts} \mathrm{s}^{-1}$ ). Like IC 3599 it was one of a few AGN that were seen during the WFC all-sky 
RASS

PSPC-pointed

\begin{tabular}{|c|c|c|c|c|c|c|c|c|c|c|c|c|}
\hline \multirow{2}{*}{ No. } & \multirow{2}{*}{ name } & \multirow[b]{2}{*}{$N_{\mathrm{H}, \mathrm{fit}}$} & \multicolumn{5}{|c|}{ RASS } & \multicolumn{5}{|c|}{ PSPC-pointed } \\
\hline & & & $\alpha_{\mathrm{x}}$ & $\chi^{2} / \nu$ & $N_{\text {H.gal }}$ & $\alpha_{\mathrm{x}}$ & $\chi^{2} / \nu$ & $\log F_{\mathrm{X}}$ & $\alpha_{\mathrm{x}}$ & $\chi^{2} / \nu$ & $\log F_{\mathrm{X}}$ & comments \\
\hline 1 & Mkn 335 & $4.49 \pm 1.36$ & $2.29 \pm 0.29$ & $15 / 19$ & 3.96 & $2.10 \pm 0.10$ & $16 / 20$ & -13.40 & $2.13 \pm 0.01$ & $233 / 178$ & -13.47 & \\
\hline 2 & ESO 242-G8 & $0.70 \pm 1.14$ & $1.13 \pm 0.66$ & $15 / 10$ & 1.48 & $1.56 \pm 0.26$ & $17 / 11$ & -14.31 & $1.64 \pm 0.05$ & $53 / 56$ & -14.45 & \\
\hline 3 & WPVS 007 & $0.68 \pm 0.71$ & $3.10 \pm 0.99$ & $5.4 / 13$ & 2.82 & $8.70 \pm 1.75$ & $8.9 / 14$ & -14.27 & - & - & - & 1 \\
\hline 4 & RX J0057-22 & $1.37 \pm 0.51$ & $1.82 \pm 0.27$ & $25 / 25$ & 1.48 & $1.89 \pm 0.10$ & $25 / 26$ & -13.77 & $1.99 \pm 0.03$ & $78 / 93$ & -13.63 & \\
\hline 5 & QSO 0056-36 & $1.73 \pm 1.19$ & $1.62 \pm 0.51$ & $29 / 22$ & 1.94 & $1.72 \pm 0.16$ & $29 / 23$ & -14.29 & - & - & - & \\
\hline 6 & RX J0100-51 & $2.49 \pm 1.59$ & $1.75 \pm 0.52$ & $16 / 18$ & 2.42 & $1.73 \pm 0.18$ & $16 / 19$ & -13.99 & - & - & - & \\
\hline 7 & RX J0105-14 & $2.34 \pm 0.91$ & $1.53 \pm 0.30$ & $30 / 19$ & 1.77 & $1.29 \pm 0.09$ & $32 / 20$ & -13.89 & - & - & - & \\
\hline 8 & RX J0117-38 & $2.82 \pm 1.35$ & $2.41 \pm 0.44$ & $3.9 / 9$ & 2.08 & $2.09 \pm 0.15$ & $5.4 / 10$ & -14.18 & - & - & - & \\
\hline 9 & MS 0117-28 & $2.13 \pm 1.56$ & $2.51 \pm 0.66$ & $6.8 / 13$ & 1.65 & $2.27 \pm 0.26$ & $7.2 / 14$ & -14.54 & $2.70 \pm 0.12$ & $28 / 31$ & -14.46 & \\
\hline 10 & RX J0128-18 & $1.82 \pm 1.31$ & $1.64 \pm 0.55$ & $14 / 19$ & 1.62 & $1.55 \pm 0.17$ & $14 / 20$ & -14.26 & - & - & - & \\
\hline 11 & IRAS01267-21 & $1.51 \pm 1.26$ & $1.55 \pm 0.62$ & $14 / 15$ & 1.28 & $1.43 \pm 0.19$ & $14 / 16$ & -14.29 & - & - & - & \\
\hline 12 & RX J0148-27 & $2.54 \pm 0.82$ & $2.62 \pm 0.30$ & $21 / 22$ & 1.50 & $2.12 \pm 0.11$ & $30 / 23$ & -13.90 & $1.88 \pm 0.03$ & $178 / 110$ & -13.90 & \\
\hline 13 & RX J0152-23 & $1.25 \pm 0.68$ & $1.75 \pm 0.39$ & $17 / 13$ & 1.10 & $1.67 \pm 0.13$ & $17 / 14$ & -14.26 & - & - & - & \\
\hline 14 & Mkn 1044 & $3.99 \pm 1.38$ & $2.04 \pm 0.32$ & $21 / 17$ & 3.16 & $1.74 \pm 0.10$ & $22 / 18$ & -13.52 & $1.75 \pm 0.03$ & $144 / 118$ & -13.52 & \\
\hline 15 & Mkn 1048 & $3.18 \pm 1.62$ & $1.67 \pm 0.43$ & $13 / 9$ & 2.83 & $1.53 \pm 0.14$ & $13 / 10$ & -13.84 & $1.51 \pm 0.03$ & $181 / 140$ & -13.77 & \\
\hline 16 & RX J0311-20 & $1.02 \pm 1.15$ & $8.63 \pm 0.56$ & $23 / 17$ & 2.37 & $1.47 \pm 0.19$ & $27 / 18$ & -14.09 & - & - & - & \\
\hline 17 & RX J0319-26 & $0.52 \pm 1.09$ & $1.39 \pm 0.73$ & $6.3 / 7$ & 1.32 & $1.79 \pm 0.31$ & $7.1 / 8$ & -14.55 & $1.42 \pm 0.03$ & $88 / 83$ & -14.23 & \\
\hline 18 & RX J0323-49 & $2.45 \pm 0.85$ & $2.35 \pm 0.29$ & $36 / 21$ & 1.72 & $2.03 \pm 0.10$ & $39 / 22$ & -14.02 & $1.83 \pm 0.05$ & $79 / 55$ & -14.39 & \\
\hline 19 & ESO 301-G13 & $1.66 \pm 0.91$ & $1.75 \pm 0.41$ & $20 / 11$ & 2.19 & $2.01 \pm 0.14$ & $21 / 12$ & -14.09 & - & - & - & \\
\hline 20 & VCV0331-37 & $1.48 \pm 1.76$ & $1.51 \pm 0.89$ & $6.0 / 7$ & 1.63 & $1.59 \pm 0.27$ & $6.0 / 8$ & -14.32 & - & - & - & \\
\hline 21 & RX J0349-47 & $0.78 \pm 0.80$ & $2.04 \pm 0.58$ & $13 / 12$ & 1.44 & $2.45 \pm 0.33$ & $15 / 13$ & -14.75 & - & - & - & \\
\hline 22 & Fair 1116 & $2.43 \pm 1.58$ & $1.87 \pm 0.53$ & $20 / 17$ & 3.84 & $2.48 \pm 0.20$ & $22 / 18$ & -13.56 & - & - & - & \\
\hline 23 & CTS 44 & $1.08 \pm 0.39$ & $1.33 \pm 0.20$ & $27 / 35$ & 1.24 & $1.41 \pm 0.07$ & $27 / 36$ & -13.66 & - & - & - & \\
\hline 24 & RX J0412-47 & $0.82 \pm 1.39$ & $1.36 \pm 0.81$ & $2.8 / 6$ & 1.42 & $1.66 \pm 0.31$ & $3.2 / 7$ & -14.34 & - & - & - & \\
\hline 25 & RX J0426-57 & $1.12 \pm 0.31$ & $1.62 \pm 0.20$ & $44 / 30$ & 2.25 & $2.20 \pm 0.07$ & $70 / 31$ & -13.53 & $2.10 \pm 0.02$ & $333 / 154$ & -13.19 & \\
\hline 26 & Fair 303 & $1.69 \pm 1.12$ & $1.89 \pm 0.51$ & $13 / 20$ & 0.99 & $1.51 \pm 0.17$ & $15 / 21$ & -14.19 & $1.31 \pm 0.05$ & $58 / 53$ & -14.24 & \\
\hline 27 & RX J0437-47 & $1.07 \pm 0.46$ & $1.74 \pm 0.31$ & $28 / 21$ & 1.69 & $2.09 \pm 0.12$ & $33 / 22$ & -14.14 & $1.93 \pm 0.03$ & $184 / 91$ & -13.96 & \\
\hline 28 & RX J0439-53 & $1.35 \pm 1.85$ & $2.28 \pm 1.04$ & $3.1 / 5$ & 1.55 & $2.39 \pm 0.42$ & $32 / 6$ & -14.44 & - & - & - & \\
\hline 29 & Н $0439-272$ & $1.98 \pm 3.99$ & $1.30 \pm 1.57$ & $6.8 / 3$ & 2.43 & $1.51 \pm 0.39$ & $6.8 / 4$ & -14.26 & $1.27 \pm 0.04$ & $90 / 88$ & -14.04 & \\
\hline 30 & 1 ES 0614-58 & $4.37 \pm 1.00$ & $2.38 \pm 0.23$ & $20 / 30$ & 4.60 & $2.46 \pm 0.08$ & $20 / 31$ & -13.90 & - & - & - & \\
\hline 31 & RX J0859+48 & $1.99 \pm 1.46$ & $1.44 \pm 0.57$ & $16 / 17$ & 2.05 & $1.46 \pm 0.17$ & $16 / 18$ & -14.22 & - & - & - & \\
\hline 32 & RX J0902-07 & $3.54 \pm 2.45$ & $2.12 \pm 0.62$ & $16 / 13$ & 3.68 & $2.17 \pm 0.22$ & $16 / 14$ & -14.08 & - & - & - & \\
\hline 33 & Mkn 110 & $1.47 \pm 0.64$ & $1.25 \pm 0.30$ & $13 / 21$ & 1.56 & $1.29 \pm 0.09$ & $13 / 22$ & -13.86 & $1.47 \pm 0.01$ & $290 / 182$ & -13.26 & \\
\hline 34 & PG $0953+41$ & $2.30 \pm 1.26$ & $2.26 \pm 0.46$ & $7.6 / 8$ & 1.14 & $1.65 \pm 0.15$ & $13 / 9$ & -14.34 & $1.55 \pm 0.04$ & $47 / 73$ & -14.28 & \\
\hline 35 & RX J1005+43 & $1.65 \pm 0.96$ & $2.15 \pm 0.46$ & $9.4 / 9$ & 1.08 & $1.81 \pm 0.16$ & $11 / 10$ & -14.49 & - & - & - & \\
\hline 36 & $\mathrm{RX} \mathrm{J1007+22}$ & $6.20 \pm 2.79$ & $2.91 \pm 0.54$ & $15 / 21$ & 2.69 & $1.68 \pm 0.16$ & $24 / 22$ & -14.19 & - & - & - & \\
\hline 37 & CBS 126 & $1.36 \pm 0.62$ & $1.62 \pm 0.33$ & $18 / 19$ & 1.41 & $1.65 \pm 0.10$ & $18 / 20$ & -14.03 & - & - & - & \\
\hline 38 & HS $1019+37$ & $1.32 \pm 0.95$ & $9.83 \pm 0.43$ & $12 / 9$ & 1.28 & $0.98 \pm 0.12$ & $12 / 10$ & -14.23 & - & - & - & \\
\hline 39 & Mkn 141 & $1.68 \pm 1.30$ & $1.84 \pm 0.58$ & $15 / 16$ & 1.07 & $1.53 \pm 0.18$ & $16 / 17$ & -14.54 & - & - & - & \\
\hline 40 & Mkn 142 & $1.61 \pm 0.55$ & $2.10 \pm 0.27$ & $34 / 26$ & 1.18 & $1.88 \pm 0.10$ & $36 / 27$ & -14.04 & $1.77 \pm 0.03$ & $139 / 85$ & -14.20 & \\
\hline
\end{tabular}




\begin{tabular}{|c|c|c|c|c|c|c|c|c|c|c|c|c|}
\hline \multirow{2}{*}{ No. } & \multirow{2}{*}{ name } & \multirow[b]{2}{*}{$N_{\mathrm{H}, \mathrm{fit}}$} & \multicolumn{5}{|c|}{ RASS } & \multicolumn{5}{|c|}{ PSPC-pointed } \\
\hline & & & $\alpha_{\mathrm{x}}$ & $\chi^{2} / \nu$ & $N_{\text {H.gal }}$ & $\alpha_{\mathrm{x}}$ & $\chi^{2} / \nu$ & $\log F_{\mathrm{X}}$ & $\alpha_{\mathrm{x}}$ & $\chi^{2} / \nu$ & $\log F_{\mathrm{X}}$ & comments \\
\hline 41 & RX J1034+39 & $2.71 \pm 0.79$ & $3.00 \pm 0.31$ & $13 / 25$ & 1.50 & $2.38 \pm 0.11$ & $26 / 26$ & -13.85 & $2.42 \pm 0.03$ & $376 / 91$ & -13.67 & \\
\hline 42 & RX J1117+65 & $1.95 \pm 1.10$ & $2.50 \pm 0.49$ & $6.9 / 9$ & 0.91 & $1.89 \pm 0.17$ & $12 / 10$ & -14.62 & - & - & - & \\
\hline 43 & PG $1115+407$ & $1.42 \pm 1.27$ & $1.81 \pm 0.66$ & $13 / 13$ & 1.93 & $2.05 \pm 0.24$ & $13 / 14$ & -14.43 & $1.98 \pm 0.06$ & $49 / 44$ & -14.53 & \\
\hline 44 & Ton 1388 & $1.14 \pm 0.91$ & $1.61 \pm 0.57$ & $11 / 7$ & 1.22 & $1.65 \pm 0.17$ & $11 / 8$ & -14.29 & $1.62 \pm 0.02$ & $193 / 149$ & -14.12 & \\
\hline 45 & EXO 1128+69 & $1.12 \pm 0.40$ & $1.49 \pm 0.22$ & $42 / 34$ & 1.34 & $1.60 \pm 0.07$ & $42 / 35$ & -13.97 & - & - & - & \\
\hline 46 & B2 $1128+31$ & $2.61 \pm 2.08$ & $1.59 \pm 0.66$ & $12 / 12$ & 2.22 & $1.43 \pm 0.20$ & $12 / 13$ & -14.38 & - & - & - & \\
\hline 47 & SBS $1136+579$ & $1.42 \pm 1.22$ & $1.77 \pm 0.63$ & $18 / 16$ & 1.00 & $1.54 \pm 0.19$ & $18 / 17$ & -14.54 & $1.58 \pm 0.19$ & $4.1 / 6$ & -15.38 & \\
\hline 48 & Z $1136+3412$ & $1.81 \pm 1.24$ & $1.72 \pm 0.52$ & $19 / 19$ & 2.04 & $1.81 \pm 0.17$ & $19 / 20$ & -14.14 & $1.81 \pm 0.09$ & $22 / 24$ & -14.36 & \\
\hline 49 & Was 26 & $2.75 \pm 0.97$ & $1.69 \pm 0.29$ & $22 / 20$ & 2.10 & $1.43 \pm 0.09$ & $24 / 21$ & -13.82 & $1.43 \pm 0.03$ & $121 / 92$ & -13.98 & \\
\hline 50 & CASG 855 & $1.64 \pm 1.03$ & $1.33 \pm 0.47$ & $9.4 / 9$ & 1.80 & $1.40 \pm 0.13$ & $9.5 / 10$ & -13.95 & - & - & - & \\
\hline 51 & Mkn 1310 & $2.31 \pm 1.37$ & $1.35 \pm 0.47$ & $14 / 8$ & 2.43 & $1.39 \pm 0.14$ & $14 / 9$ & -14.01 & $1.13 \pm 0.12$ & $18 / 14$ & -14.71 & \\
\hline 52 & NGC 4051 & $2.23 \pm 0.59$ & $2.02 \pm 0.22$ & $31 / 36$ & 1.37 & $1.62 \pm 0.07$ & $41 / 37$ & -13.60 & $1.65 \pm 0.01$ & $361 / 177$ & -13.92 & \\
\hline 53 & GQ Com & $1.94 \pm 1.23$ & $1.30 \pm 0.50$ & $27 / 21$ & 1.66 & $1.18 \pm 0.15$ & $27 / 22$ & -14.37 & $1.20 \pm 0.02$ & $131 / 140$ & -14.40 & \\
\hline 54 & RX J1209+32 & $4.34 \pm 4.52$ & $3.18 \pm 1.18$ & $2.7 / 5$ & 0.00 & $0.86 \pm 0.27$ & $19 / 6$ & -14.73 & $1.09 \pm 0.09$ & $36 / 20$ & -14.98 & \\
\hline 55 & PG $1211+143$ & $3.04 \pm 1.02$ & $2.12 \pm 0.29$ & $22 / 21$ & 2.75 & $2.00 \pm 0.09$ & $2.2 / 22$ & -13.74 & $1.82 \pm 0.04$ & $84 / 78$ & -13.86 & \\
\hline 56 & Mkn 766 & $3.40 \pm 0.64$ & $1.77 \pm 0.16$ & $19 / 29$ & 1.69 & $1.11 \pm 0.05$ & $57 / 30$ & -13.31 & $1.28 \pm 0.01$ & $474 / 171$ & -13.48 & \\
\hline 57 & $3 \mathrm{C} 273$ & $1.58 \pm 0.34$ & $1.21 \pm 0.16$ & $56 / 42$ & 1.79 & $1.30 \pm 0.05$ & $57 / 43$ & -13.23 & $1.00 \pm 0.01$ & $234 / 200$ & -13.18 & \\
\hline 58 & RX J1231+70 & $1.95 \pm 0.75$ & $1.49 \pm 0.29$ & $16 / 22$ & 1.67 & $1.38 \pm 0.08$ & $16 / 23$ & -14.12 & - & - & - & \\
\hline 59 & Mkn 771 & $3.64 \pm 1.88$ & $2.46 \pm 0.51$ & $19 / 19$ & 2.05 & $1.83 \pm 0.16$ & $24 / 20$ & -14.32 & $1.64 \pm 0.03$ & $123 / 126$ & -13.87 & \\
\hline 60 & CBS 150 & $3.41 \pm 2.01$ & $2.94 \pm 0.62$ & $20 / 18$ & 1.44 & $2.13 \pm 0.20$ & $26 / 19$ & -14.71 & $1.89 \pm 0.09$ & $23 / 27$ & -14.60 & \\
\hline 61 & $\mathrm{MCG}+08-23-06$ & $1.36 \pm 1.09$ & $1.36 \pm 0.53$ & $19 / 19$ & 1.40 & $1.38 \pm 0.16$ & $19 / 20$ & -14.37 & - & - & - & \\
\hline 62 & IC 3599 & $4.04 \pm 0.71$ & $3.37 \pm 0.21$ & $55 / 32$ & 1.34 & $2.17 \pm 0.06$ & $169 / 33$ & -13.60 & $2.61 \pm 0.35$ & $11 / 6$ & -15.59 & \\
\hline 63 & NGC 4593 & $1.57 \pm 0.94$ & $1.19 \pm 0.42$ & $15 / 11$ & 2.28 & $1.47 \pm 0.12$ & $16 / 12$ & -13.46 & $1.19 \pm 0.05$ & $54 / 54$ & -13.78 & \\
\hline 64 & IRAS $1239+33$ & $2.74 \pm 1.38$ & $2.02 \pm 0.42$ & $19 / 11$ & 1.35 & $1.37 \pm 0.12$ & $25 / 12$ & -14.24 & $1.28 \pm 0.03$ & $195 / 98$ & -14.43 & \\
\hline 65 & PG $1244+026$ & $1.08 \pm 0.99$ & $1.44 \pm 0.53$ & $21 / 16$ & 1.75 & $1.79 \pm 0.20$ & $22 / 17$ & -14.06 & $1.85 \pm 0.03$ & $118 / 83$ & -14.02 & \\
\hline 66 & RX J1304+02 & $1.50 \pm 1.13$ & $2.26 \pm 0.59$ & $18 / 18$ & 1.74 & $2.38 \pm 0.25$ & $18 / 19$ & -14.44 & - & - & - & \\
\hline 67 & PG $1307+085$ & $2.75 \pm 1.85$ & $1.87 \pm 0.55$ & $8.8 / 17$ & 2.05 & $1.58 \pm 0.17$ & $8.9 / 18$ & -14.32 & $1.58 \pm 0.04$ & $86 / 80$ & -14.24 & \\
\hline 68 & RX J1314+34 & $1.93 \pm 1.08$ & $2.35 \pm 0.45$ & $9.5 / 9$ & 0.99 & $1.88 \pm 0.16$ & $13 / 10$ & -14.51 & $1.76 \pm 0.14$ & $24 / 11$ & -14.87 & \\
\hline 69 & RX J1319+52 & $2.04 \pm 1.12$ & $2.06 \pm 0.44$ & $4.5 / 9$ & 1.16 & $1.60 \pm 0.14$ & $8.0 / 10$ & -14.41 & - & - & - & \\
\hline 70 & PG $1322+659$ & $1.32 \pm 0.77$ & $1.74 \pm 0.42$ & $5.5 / 10$ & 2.01 & $2.07 \pm 0.15$ & $7.5 / 11$ & -14.30 & $1.81 \pm 0.04$ & $64 / 73$ & -14.27 & \\
\hline 71 & IRAS $1334+24$ & $1.36 \pm 0.50$ & $2.01 \pm 0.27$ & $20 / 25$ & 1.12 & $1.88 \pm 0.10$ & $21 / 26$ & -13.92 & $1.83 \pm 0.05$ & $57 / 53$ & -14.20 & \\
\hline 72 & Ton 730 & $1.29 \pm 0.99$ & $1.96 \pm 0.57$ & $13 / 16$ & 1.05 & $1.83 \pm 0.20$ & $13 / 17$ & -14.59 & - & - & - & \\
\hline 73 & RX J1355+56 & $1.85 \pm 0.92$ & $2.31 \pm 0.42$ & $15 / 10$ & 1.15 & $1.93 \pm 0.15$ & $18 / 11$ & -14.47 & - & - & - & \\
\hline 74 & PG $1402+25$ & $1.52 \pm 1.01$ & $1.85 \pm 0.50$ & $4.0 / 7$ & 1.48 & $1.83 \pm 0.17$ & $4.0 / 8$ & -14.42 & $1.91 \pm 0.06$ & $51 / 49$ & -14.12 & \\
\hline 75 & RX J1413+70 & $1.15 \pm 0.98$ & $1.07 \pm 0.47$ & $13 / 23$ & 1.93 & $1.40 \pm 0.15$ & $14 / 24$ & -14.20 & - & - & - & \\
\hline 76 & NGC 5548 & $1.47 \pm 0.38$ & $1.13 \pm 0.17$ & $47 / 30$ & 1.93 & $1.33 \pm 0.05$ & $52 / 31$ & -13.35 & $1.50 \pm 0.01$ & $324 / 201$ & -13.10 & \\
\hline 77 & Q 1421-0013 & $5.99 \pm 3.21$ & $2.71 \pm 0.62$ & $17 / 14$ & 2.97 & $1.72 \pm 0.19$ & $22 / 15$ & -14.23 & - & - & - & \\
\hline 78 & Mkn 813 & $1.29 \pm 0.87$ & $1.11 \pm 0.42$ & $8.2 / 10$ & 2.54 & $1.64 \pm 0.14$ & $13 / 11$ & -14.12 & - & - & - & \\
\hline 79 & Mkn 684 & $1.68 \pm 1.38$ & $1.44 \pm 0.61$ & $12 / 15$ & 1.50 & $1.36 \pm 0.18$ & $13 / 16$ & -14.40 & - & - & - & \\
\hline 80 & Mkn 478 & $1.27 \pm 0.26$ & $2.22 \pm 0.16$ & $20 / 32$ & 1.04 & $2.08 \pm 0.06$ & $24 / 33$ & -13.64 & $2.18 \pm 0.05$ & $68 / 54$ & -13.95 & \\
\hline
\end{tabular}




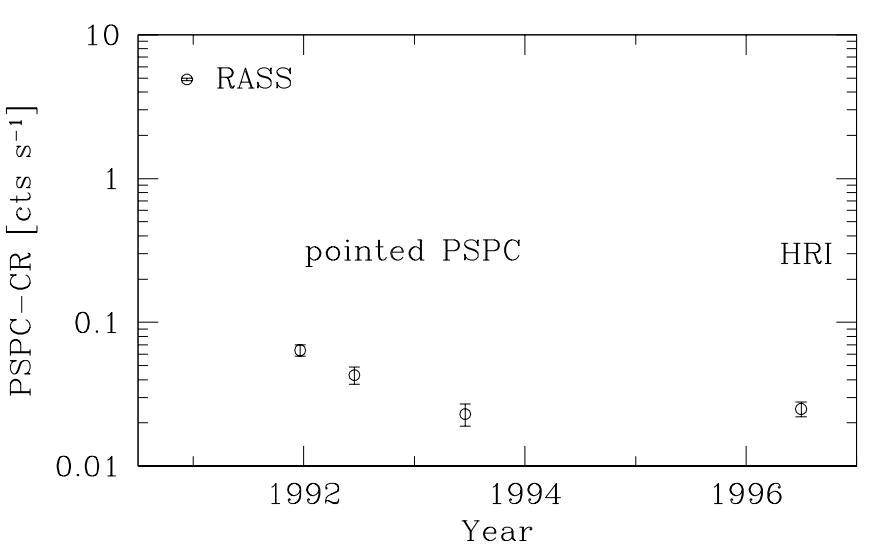

Fig. 6. Long-term light curve of IC 3599. The HRI count rate was converted into a PSPC count rate (see Sect. 2)

Table 5. Pointed HRI observations of WPVS007. The UT observing dates are given as yymmdd, the exposure time is in $\mathrm{s}$, the HRI count rate in units of $10^{-4} \mathrm{HRI} \mathrm{cts} \mathrm{s}^{-1}$, and the converted PSPC count rate in units of $10^{-3} \mathrm{PSPC} \operatorname{cts~s}^{-1} .3 \sigma$ Upper limits are marked as (u.l.)

\begin{tabular}{cccrcc}
\hline$\#$ & ROR & obs. date & $T_{\text {obs }}$ & HRI CR & PSPC CR \\
\hline 1 & 702703 & 951118 & 15994 & $4.04($ u.l.) & 3.22 \\
2 & 702705 & 960613 & 19863 & $5.14 \pm 3.48$ & 4.10 \\
3 & 702923 & 971110 & 10851 & $4.61 \pm 4.33$ & 3.68 \\
4 & 702921 & 971206 & 9976 & $5.20($ u.l) & 4.16 \\
& all & - & 56684 & $5.29 \pm 2.00$ & 4.22 \\
\hline
\end{tabular}

survey (Edelson et al. 1999). After the RASS it was observed three years later by the ROSAT PSPC and was practically "turned-off". In the following years we monitored the source four times (see Table 5) using the ROSAT HRI. In two of these observations WPVS007 was detected again (ROR 702705 and 702923). For the other two only upper limits can be given. Figure 7 shows the new longterm light curve of WPVS007. The HRI count rates of WPVS007 convert to about $\log L_{\mathrm{X}}=31$ [W]. Like IC 3599 we monitored WPVS007's optical spectrum over several years. So far we not detected any change in the optical spectrum. A possible interpretation of the transience in WPVS007 is that the temperature of the Comptonization layer above the disk that scatters the thermal UV disk photons into the soft X-ray range, changed. A lower temperature caused a shift of the EUV bump spectrum out of the ROSAT energy range of $0.1-2.4 \mathrm{keV}$. This explains why the bolometric luminosity changed by a factor of 2 but the PSPC count rate decreased by a factor of 400 (Grupe et al. 1995b). It is also interesting to note that WPVS007 was detected by the HRI considering that no hard photons above $0.5 \mathrm{keV}$ were detected in the RASS observation $^{4}$.

\footnotetext{
4 The HRI has a lower efficiency in soft X-rays than the PSPC.
}

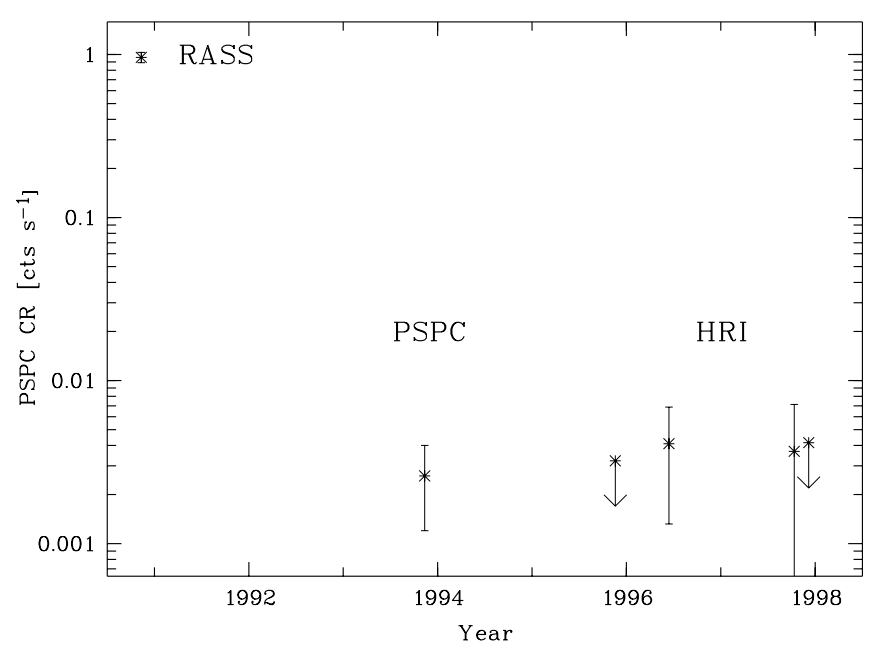

Fig. 7. Long-term light curve of WPVS007. The downwards arrows mark the upper limits of the HRI observations converted into PSPC counts (see Sect. 2)

\subsubsection{RX J1624.9+7554}

This X-ray transient was discovered in a non-active spiral galaxy (Grupe et al. 1999b). Between the RASS and the pointed PSPC observations years later the object vanished completely in X-rays. Because this source is in its normal state a non-active galaxy, it is most likely that its X-ray outburst was caused by a tidal disruption of a star by the central black hole. Komossa \& Greiner (1999) and most recently Greiner et al. (2000) also reported about other $\mathrm{X}$-ray outbursts in a non-active galaxies.

\subsubsection{RX J2217.9-5941}

This Narrow-line Seyfert 1 galaxy has shown a decrease in its count rate by a factor of about 30 between the RASS and its last HRI observation in 1998 (Grupe et al. 2001). It is not yet clear if this is a real transient source, which means the the count rate is still decreasing unti today, or if it was observed in its "low-state" in both HRI observations in 1997 and 1998. The RASS light curve shows a decline in count rate by a factor of approximately 15 in about two days. However, the HRI count rates do not show strong variability over their four day coverage.

\subsubsection{RX J2349.3-3126}

RX J2349.3-3126 is the only source in the current sample that has shown a significant change in its X-ray spectrum between the RASS and its pointed PSPC observation two and a half years later. Its hardness ratio has changed from $-0.53 \pm 0.06$ during the RASS to $-0.21 \pm 0.13$ in the pointed observation (Table 1). It also showed a decrease in its mean count rate between the RASS and the pointed observation by a factor of about 6. RX J2349.3-3126 seems to show large amplitude variations only over long time scales. During the RASS coverage the source doubled its count rate in about two days. During the pointed 
PSPC observation the source remained almost constant over about 6 hours.

Optically, RX J2349.3-3126 is a Seyfert 1 galaxy with very broad emission lines $\left(F W H M(\mathrm{H} \beta)=7715 \mathrm{~km} \mathrm{~s}^{-1}\right.$; Grupe et al. 1999a). An R-image taken with the ESO/MPG $2.2 \mathrm{~m}$ telescope at La Silla besides the very bright nucleus displays several bright spots, and tidal tails. So this AGN may be fueled by a merger.

\section{Discussion}

\subsection{X-ray transient $A G N$}

Why are there no "turn-ons" among the sources of our sample? This is a selection effect due to the definition of our sample. Because our sample is chosen to include only sources that were bright during the RASS, we will be biased against sources that were faint then but have since brightened, but we include sources that have since become fainter - sometimes much fainter - than our original count rate limit. For obvious reasons, only the brightest sources were re-observed in later pointed observations. Fainter RASS sources can be seen only serendipitously brighter in pointed observations. Because of the much smaller coverage of the sky in the pointed observations (15\% PSPC, $2 \%$ HRI; Voges, private communications) there is not much chance to detect a transient in its "high" state. So far only one AGN has been reported being faint in the RASS and bright in a later pointed PSPC observation, RX J1242.61119 (Komossa \& Greiner 1999).

What causes transience in AGN? The most common explanation of X-ray transience in AGN is a sudden increase of the accretion rate. This could be the result of either accretion disk instabilities, or even the tidal disruption of a star as has been suggested by Rees (1990). While in the Seyfert 2 galaxy IC 3599 both accretion disk instabilities or a tidal disruption of a star might cause the X-ray outburst (Brandt et al. 1995; Grupe et al. 1995a) in non active galaxies like RX J1624.9+7554, RX J1242.6-1119, NGC 5905 (Komossa \& Bade 1999) or RX J1420.4+5334 (Greiner et al. 2000) tidal disruption seems to be the more likely explanation. In the case of the X-ray transient WPVS007, Grupe et al. (1995b) discussed the possibility of a temperature change in the Comptonization layer above the disk as an alternative explanation. A lower temperature of this layer would shift the soft X-ray spectrum out of the ROSAT PSPC energy window $(0.1-2.4 \mathrm{keV})$ and would mimic a dramatic change in the X-ray/Big Blue Bump flux.

Converting the HRI count rates of the two transients IC 3599 and WPVS007 into X-ray fluxes shows that they now have typical X-ray luminosities of a normal galaxy (about $\log L_{\mathrm{X}}=33[\mathrm{~W}]$ ). In both cases, we can consider this as their pre-outburst/transient luminosities.

\subsection{X-ray variability}

We find that low-luminosity objects have a higher probability of being found to be variable than the highluminosity ones (Fig. 2). This result is not as prominent as in the samples of Boller et al. (1996) and Leighly (1999a,b). The reason is that their samples stretch over 7 and 5 orders in luminosity while ours only stretches over $\approx 2$ orders. However, in our Principal Component Analysis on our original sample (Grupe et al. 1998a, 1999a) we found this result as a part of the Eigenvector 1 relationship. There are two explanations for the dependence of the variability on luminosity: a) the size of the Black Hole/AGN engine, and b) the number of the X-ray emitting regions (see discussion in Leighly 1999a). The $\chi^{2} / \nu$ test for the short-term variability in the RASS data is a robust test. It does not take the length of the observation into account. However, the RASS coverages are usually in the order of days and therefore comparable. Only for a few sources the RASS observations were split into two parts half a year apart. We should mention that the result of the distribution of the variability seen in Fig. 2 is smeared out when the excess variance (e.g. Nandra et al. 1997; Leighly 1999a) is used instead if $\chi^{2} / \nu$.

The comparison of the long-term variability (right panel of Fig. 2) is more complicated. The time gaps between RASS and pointed observation can vary from source to source between half a year up to 8 years. This is the reason why the HRI data points suggest less variability than the PSPC data, because they have been observed later than the PSPC. The only way to check out the long term variability of a large sample of AGN is repeated monitoring of the sky like it is performed for example by RXTE's All-Sky Monitor.

Figure 3 in Leighly 1999a shows that NLS1 are more variable than Broad-line Seyfert 1s. In our sample a large percentage are NLS1 (Grupe et al. 1999a; Grupe et al. 2001, in prep). NLS1 are also objects that have the steepest X-ray spectra (Boller et al. 1996; Grupe et al. 1998a). Therefore we checked for a relation between $\alpha_{\mathrm{x}}$ and the strength of the variability. This is what we find for the short-term variability throughout the sample: objects with steeper X-ray spectra (preferentially NLS1) show stronger variability than those with flatter X-ray spectra (see Fig. 3). This is in agreement with the findings of Green et al. (1993) where "sources with steeper energy spectra have higher normalized variability amplitudes".

In principle, the same intrinsic processes that apply to $\mathrm{X}$-ray transience (changes in the accretion rate or the disk temperature) also apply to the normal variability, but on a much lower level. In cases of very rapid variability, such as found in IRAS 13224-3809 (Boller et al. 1997), relativistic and Doppler boosting and gravitational lensing effects (see Boller et al. 1997; Leighly 1999a) have to be taken into account. The variability will be stronger amplified in steep X-ray sources.

Another alternative explanation of variability is a change in the cold and warm absorber column densities 
and their ionization states (e.g. Abrassart \& Czerny 2000; Komossa \& Meerschweinchen 2000).

We have shown that ROSAT with its All-Sky Survey and the later pointed observations was a well suited experiment to detect X-ray transient sources and to monitor the long-term behaviour of AGN. The best way to find more transients would be to perform all-sky surveys repeatedly.

Acknowledgements. We thank Drs Bev Wills, Mario Gliozzi, Wolfgang Voges, Stefanie Komossa, and Joachim Trümper for useful suggestions and discussions. We also want to thank our referee, Prof. Dr. A. Lawrence for his comments on the manuscript and valuable information on additional references. This research has made use of the NASA/IPAC Extragalactic Database (NED) which is operated by the Jet Propulsion Laboratory, Caltech, under contract with the National Aeronautics and Space Administration. The ROSAT project is supported by the Bundesministerium für Bildung, Wissenschaft, Forschung und Technologie (BMBF) and the Max-Planck-Gesellschaft.

This paper can be retrieved via WWW: http://www . xray.mpe.mpg.de/dgrupe/research/refereed.html

\section{References}

Abrassart, A., \& Czerny, B. 2000, A\&A, 356, 475

Barr, P., \& Mushotzky, R. F. 1986, Nature, 330, 421

Boller, Th, Brandt, W. N., \& Fink, H. H. 1996, A\&A, 305, 53

Boller, Th., Brandt, W. N., Fabian, A. C., \& Fink, H. H. 1997, MNRAS, 289, 393

Boroson, T. A., \& Green, R. F. 1992, ApJS, 80, 109

Brandt, W. N., Pounds, K. A., \& Fink, H. H. 1995, MNRAS, 273, L47

Brandt, W. N., Boller, Th., Fabian, A. C., \& Ruszkowski, M. 1999, MNRAS, 303, L53

Córdova, F. A., Kartje, J. F., Thompson, R. J., et al. 1992, ApJS, 81, 661

Dickey, J. M., \& Lockman, F. J. 1990, ARA\&A, 28, 215

Edelson, R., Vaughan, S., Warwick, R., Puchnarewicz, E., \& George, I. 1999, MNRAS, 307, 91

Green, A. R., McHardy, I. M., \& Lehto, H. J. 1993, MNRAS, 265,664

Greiner, J., Schwarz, R., Zharikov, S., \& Orio, M. 2000, A\&A, $362, \mathrm{~L} 25$

Grupe, D., Beuermann, K., Mannheim, K., et al. 1995a, A\&A, 299, L5
Grupe, D., Beuermann, K., Mannheim, K., et al. 1995b, A\&A, 300, L21

Grupe, D., Beuermann, K., Mannheim, K., Thomas, H.-C., \& Fink, H. H. 1998a, A\&A, 330, 25

Grupe, D., Wills, B. J., Wills, D., \& Beuermann, K. 1998b, A\&A, 333, 827

Grupe, D., Beuermann, K., Mannheim, K., \& Thomas, H.-C. 1999a, A\&A, 350, 805

Grupe, D., Thomas, H.-C., \& Leighly, K. M. 1999b, A\&A, 350, L31

Grupe, D., Wills, B. J., \& Wills, D. 1999c, ASP Conf. Ser., 175,347

Grupe, D., Leighly, K. M., Thomas, H.-C., \& LaurentMuehleisen, S. A. 2000, A\&A, 356, 11

Grupe, D., Thomas, H.-C., \& Leighly, K. M. 2001, A\&A, accepted

Komossa, S., \& Bade, N. 1999, A\&A, 343, 775

Komossa, S., \& Greiner, J. 1999, A\&A, 349, L45

Komossa, S., \& Meerschweinchen, J. 2000, A\&A, 354, 411

Lawrence, A., Pye, J. P., \& Elvis, M. 1977, MNRAS, 181, 93P

Lawrence, A., \& Papadakis, I. 1993, ApJ, 414, L85

Leighly, K. M., Mushotzky, R. F., Yaqoob, T., Kunieda, H., \& Edelson, R. 1996, ApJ, 469, 147

Leighly, K. M. 1999a, ApJS, 125, 297

Leighly, K. M. 1999b, ApJS, 125, 317

Nandra, K., George, I. M., Mushotzky, R. F., Turner, T. J., \& Yaqoob, T. 1997, ApJ, 476, 70

Peterson, B. M., McHardy, I. M., Wilkes, B. J., et al. 2000, ApJ, in press

Pfeffermann, E., Briel, U. G., Hippmann, H., et al. 1986, SPIE, 733,519

Piro, L., Massaro, E., Perola, G. C., \& Molteni, D. 1988, ApJ, $325, \mathrm{~L} 25$

Pounds, K. A., Allan, D. J., Barber, C., et al. 1993, MNRAS, 260, 77

Puchnarewicz, E. M., Mason, K. O., Córdova, F. A., et al. 1992, MNRAS, 256, 589

Rees, M. J. 1990, Sci., 247, 817

Ross, R. R., Fabian, A. C., \& Mineshige, S. 1992, MNRAS, 258, 189

Thomas, H.-C., Beuermann, K., Reinsch, K., et al. 1998, A\&A, 335,467

Trümper, J. 1983, Adv. Space Res., 4, 241

Voges, W., Aschenbach, B., Boller, Th., et al. 1999, A\&A, 349, 389

Zimmermann, U., Boese, G., Becker, W., et al. 1998, EXSAS User's Guide, MPE Report

(http://wave.xray.mpe.mpg.de/exsas/users-guide) 